Supplement of

\title{
Atmospheric organochlorine pesticides and polychlorinated biphenyls in urban areas of Nepal: spatial variation, sources, temporal trends, and long-range transport potential
}

Balram Pokhrel et al.

Correspondence to: Xiaoping Wang (wangxp@itpcas.ac.cn)

The copyright of individual parts of the supplement might differ from the CC BY 3.0 License. 


\begin{tabular}{|c|c|c|}
\hline Text SI-1 & Description about the Study area & Page 4 \\
\hline Figure SI-1 & $\begin{array}{l}\text { Wind field of over Nepal; a. Indian monsoon (June to September) b. } \\
\text { Winter (October to January) }\end{array}$ & Page 5 \\
\hline Figure SI-2 & $\begin{array}{l}\text { Monthly average precipitation (a) and temperature variation (b) in } \\
\text { different cities }\end{array}$ & Page 6 \\
\hline Table SI-1 & $\begin{array}{l}\text { Details of PUF-PAS sampling sites in the three major cities of } \\
\text { Nepal }\end{array}$ & Page 7 \\
\hline Table SI-2 & PUF-PAS sampling time & Page 8 \\
\hline Text SI-2 & Chemical cleanup procedure & Page 8 \\
\hline Text SI-3 & Details about the gas chromatography temperature program & Page 8 \\
\hline Table SI-3 & Data of Field blanks and Method detection limits (MDL) ng/PAS & Page 9 \\
\hline Text SI-4 & Details about PUF-disk and Sampling rate & Page 10 \\
\hline Table SI-4 & Site specific sampling rate & Page 11 \\
\hline Table SI-5a & $\begin{array}{l}\text { Site specific concentrations ( } \mathrm{pg} / \mathrm{m} 3) \text { of OCPs in different urban sites } \\
\text { of Kathmandu }\end{array}$ & Page 12 \\
\hline Table SI-5b & $\begin{array}{l}\text { Site specific concentrations }(\mathrm{pg} / \mathrm{m} 3) \text { of OCPs in different urban sites } \\
\text { of Pokhara }\end{array}$ & Page 14 \\
\hline Table SI-5c & $\begin{array}{l}\text { Site specific concentrations ( } \mathrm{pg} / \mathrm{m} 3) \text { of OCPs in different urban sites } \\
\text { of Hetauda }\end{array}$ & Page 17 \\
\hline Table SI-6a & $\begin{array}{l}\text { Site specific concentrations (pg/m3) of PCBs in different urban sites } \\
\text { of Kathmandu }\end{array}$ & Page 18 \\
\hline Table SI-6b & $\begin{array}{l}\text { Site specific concentrations }(\mathrm{pg} / \mathrm{m} 3) \text { of PCBs in different urban sites } \\
\text { of Pokhara }\end{array}$ & Page 20 \\
\hline Table SI-6c & $\begin{array}{l}\text { Site specific concentrations ( } \mathrm{pg} / \mathrm{m} 3) \text { of OCPs in different urban sites } \\
\text { of Hetauda }\end{array}$ & Page 22 \\
\hline Table SI-7 & $\begin{array}{l}\text { Comparison of current levels }\left(\mathrm{pg} / \mathrm{m}^{3}\right) \text { of various POPs with different } \\
\text { tropical/subtropical urban sites }\end{array}$ & Page 23 \\
\hline Table SI-8 & $\begin{array}{l}\text { Range and average }(\mathrm{pg} / \mathrm{m} 3) \text { with Highest to lowest concentration } \\
\text { ratio }(\mathrm{H} / \mathrm{L}) \text { of different isomers/congeners }\end{array}$ & Page 24 \\
\hline Figure SI-3 & $\begin{array}{l}\text { Box and whisker plot to show distribution of different isomers of } \\
\text { DDT and its metabolites in Kathmandu, Pokhara and Hetauda }\end{array}$ & Page 25 \\
\hline Figure SI-4 & Isomers/metabolites ratios of selected OCPs to predict source type & Page 25 \\
\hline Figure SI-5 & $\begin{array}{l}\text { Box and whisker plot to show distribution of different isomers of } \\
\text { HCH and endosulfan in Kathmandu, Pokhara and Hetauda }\end{array}$ & Page 26 \\
\hline Figure SI-6 & $\begin{array}{l}\text { Box and whisker plot to show distribution of different congeners of } \\
\text { PCBs in Kathmandu, Pokhara and Hetauda }\end{array}$ & Page 26 \\
\hline Table SI-9 & $\begin{array}{l}\text { P-values (one-way ANOVA) for significant variation in levels of } \\
\text { different POPs in different sites }\end{array}$ & Page 27 \\
\hline Table SI-10 & $\begin{array}{l}\text { Significant differences }(\mathrm{P}<0.05 \text {, Tukey's Test) in OCPs } \\
\text { concentrations among the sites in Kathmandu }\end{array}$ & Page 28 \\
\hline Table SI-11 & $\begin{array}{l}\text { Significant differences }(\mathrm{P}<0.05 \text {, Tukey's post hocTest) in OCPs } \\
\text { concentrations among the sites in Hetauda }\end{array}$ & Page 28 \\
\hline Figure SI-7 & $\begin{array}{l}\text { Atmospheric level of OCPs in different land cover types in Pokhara; } \\
\text { (P1-Cropland; P2-Vegetable production and Market area; P3- } \\
\text { Industrial area; and P4- Tourist place) }\end{array}$ & Page 29 \\
\hline
\end{tabular}




\begin{tabular}{|l|l|l|}
\hline Figure SI-8 & $\begin{array}{l}\text { Atmospheric level of OCPs in different land cover types in Hetauda; } \\
\text { (H1- Crop Land; H2-Vegetable production /residential area; H3- } \\
\text { Industrial area) }\end{array}$ & Page 30 \\
\hline Figure SI-9 & $\begin{array}{l}\text { Seasonality of DDTs and HCHs in Kathmandu city (K1: Cropland, } \\
\text { K3: Industrial area, K4: Tourist area, K5: Residential area, K6: mix } \\
\text { of farm land and industrial area) }\end{array}$ & Page 31 \\
\hline Figure SI-10 & $\begin{array}{l}\text { Seasonality of DDTs and HCHs in Pokhara city (P1: Cropland, P3: } \\
\text { Industrial area, P4: Tourist area) }\end{array}$ & Page 32 \\
\hline Figure SI-11 & $\begin{array}{l}\text { Seasonality of DDTs and HCHs in Hetauda (H1: Cropland, H3: } \\
\text { Industrial area) }\end{array}$ & Page 33 \\
\hline Figure SI-12 & Seasonal variation of HCB in 3 cities of Nepal & Page 34 \\
\hline Text SI-5 & Estimation of Long range transport potential & Page 35 \\
\hline Table SI-12 & $\begin{array}{l}\text { Temperature dependent Henry's law constant and vapor pressure } \\
\text { with Rate constant of hydroxyl radical reaction at 25 }{ }^{\circ} \mathrm{C}\end{array}$ \\
\hline Table SI-13 & $\begin{array}{l}\text { Calculated values of degradation and deposition rates }\left(\mathrm{s}^{-1}\right) \text { based on } \\
\text { field temperature and precipitation }\end{array}$ & Page 37 \\
\hline Table SI-14 & $\begin{array}{l}\text { Comparison of characteristic travel distance }(\mathrm{CTD}) \mathrm{km} \text {, in current } \\
\text { study areas with global and other specified regions }\end{array}$ & Page 39 \\
\hline Text SI-6 & Uncertainties of CTD & Page 40 \\
\hline Text SI-7 & Generation of forward trajectories & Page 40 \\
\hline Figure SI-13 & Clusters of forward trajectories & Page 41 \\
\hline References & & \\
\hline
\end{tabular}


Kathmandu (1350m asl) located in mountain valley is the capital city of Nepal, with very dense population (an area of 642 sq. $\mathrm{km}$, population $=2.5$ million,). Agriculture, industry (instant food, clothes, bricks), and tourism are the major economy of Kathmandu. Pokhara (750-1050m asl) is second largest city after Kathmandu, covering an area of $225 \mathrm{sq} . \mathrm{km}$ with a population of 0.3 million. Hetauda $(100 \mathrm{~m})$ is relatively small with 135,475 populations. Different from Kathmandu, Pokhara and Hetauda are agricultural cities, with large area of crop and vegetable production place and market. Climate of these 3 cities are commonly influenced by the Indian monsoon in summer and westerlies (south branch) in winter. Summer is warm and wet particularly in July-August but winter is dry and cold. Among the cities, Hetauda is warmest followed by Pokhara and Kathmandu, whereas annual rainfall follows the order Pokhara $(\sim 3900 \mathrm{~mm})>$ Hetauda $(\sim 2250 \mathrm{~mm})>$ Kathmandu (1450mm). 


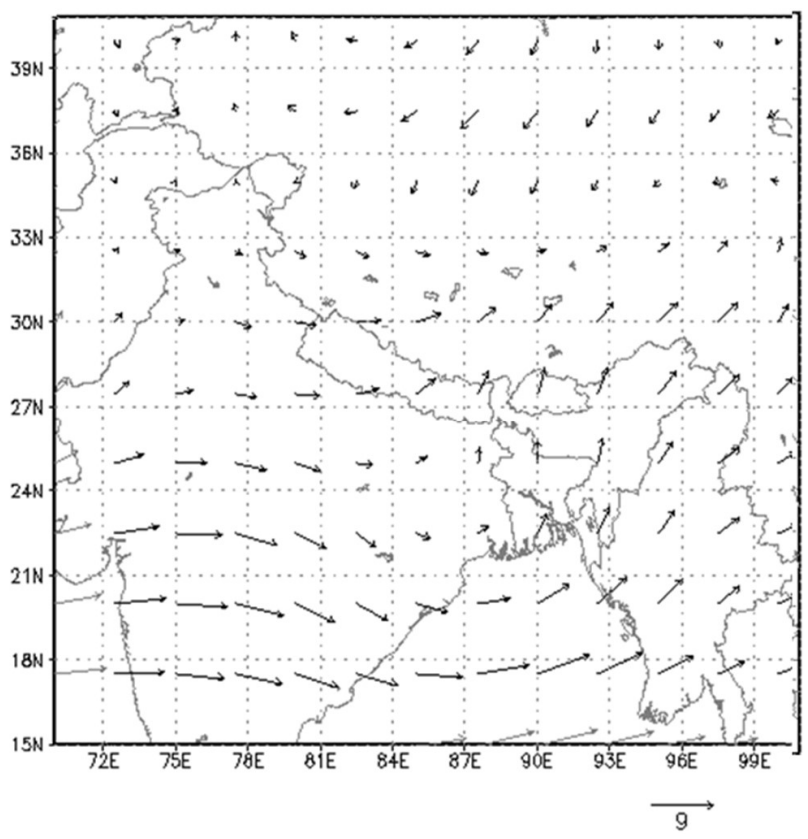

42

43

(a)

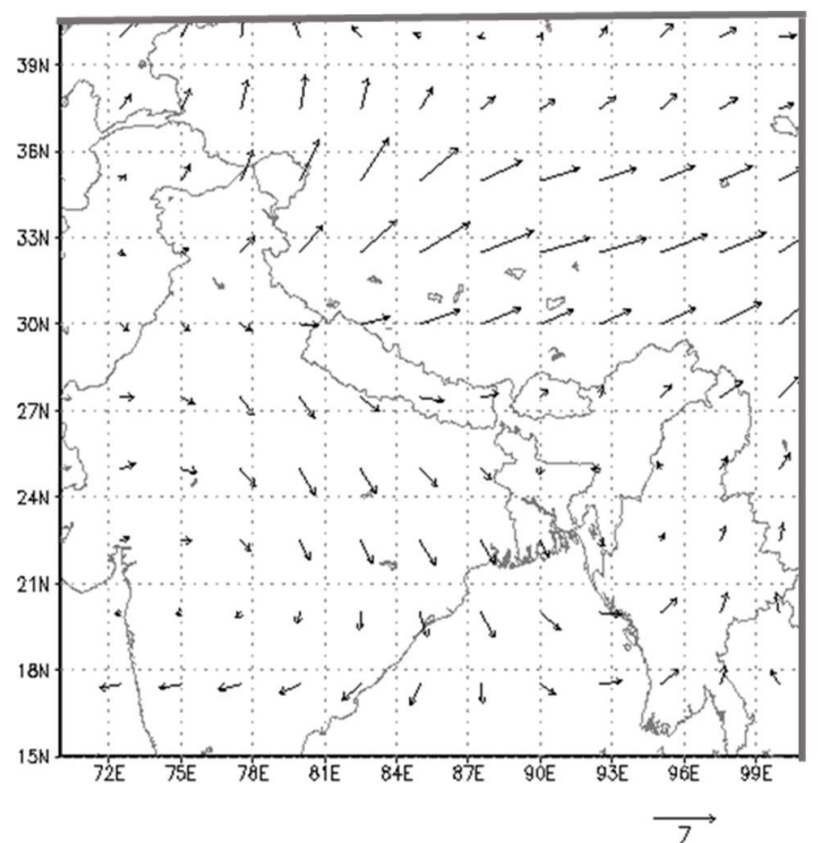

$\longrightarrow$

Figure SI-1. Wind field of over Nepal; a. Indian monsoon (June to September) b. Winter 


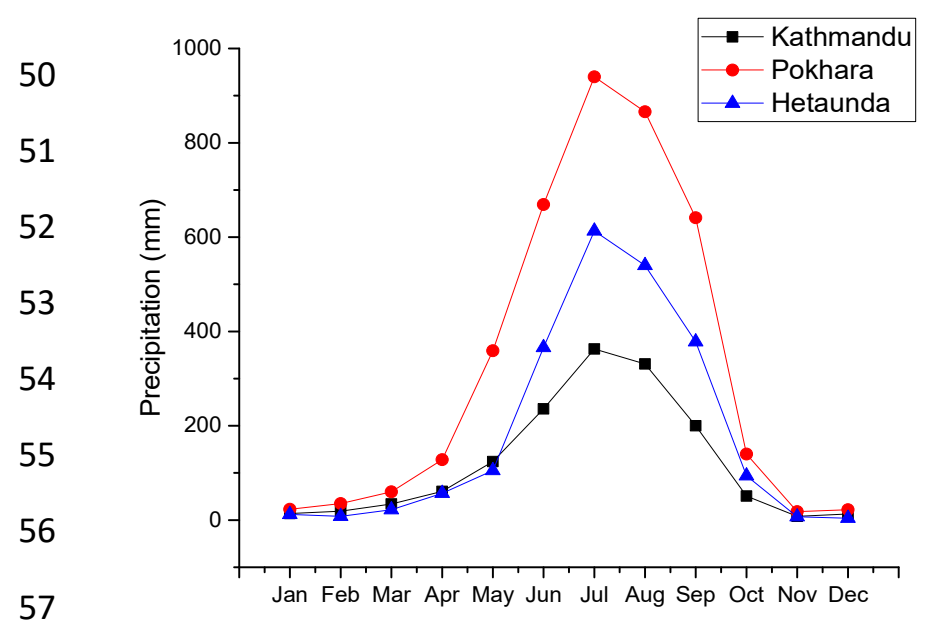

57

58

(a)

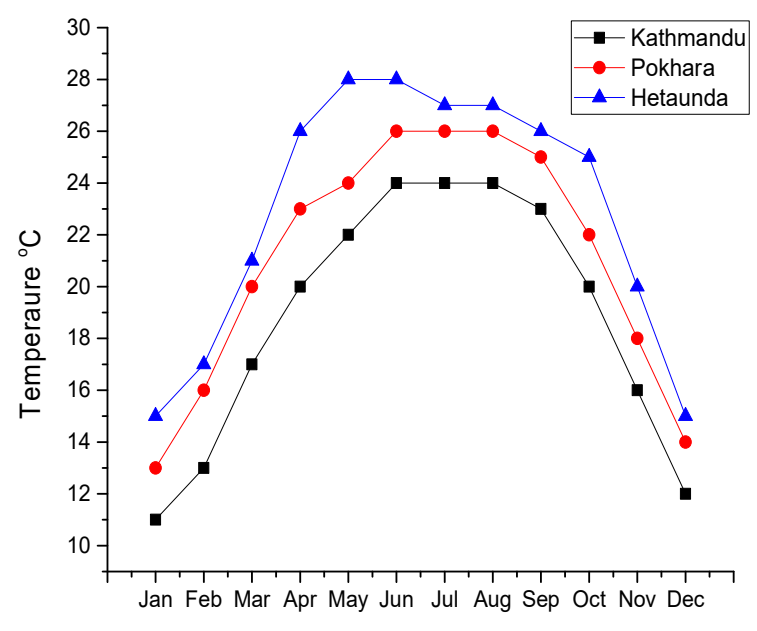

(b)

59

Figure SI-2. Monthly average of (a) precipitation and (b) temperature variation in 3 cities of 60 Nepal (Data source: Department of Hydrology and Meteorology, Nepal) 


\begin{tabular}{|c|c|c|c|c|c|}
\hline PAS & Land type & Latitude $\mathrm{N}$ & Longitude $\mathrm{E}$ & Altitude & Site description \\
\hline \multicolumn{6}{|c|}{ Kathmandu $\left(27^{\circ} 42{ }^{\prime} \mathrm{N} ; 8^{\circ} \mathbf{1 8}^{\prime} \mathrm{E}\right)$} \\
\hline K1 & Cropland & $27^{\circ} 36^{\prime} 38.92^{\prime \prime}$ & $85^{\circ} 21^{\prime} 30.79^{\prime \prime}$ & 1433 & Sub-urban site, south of the Kathmandu valley, produce maize, rice \\
\hline $\mathrm{K} 2$ & Market area & $27^{\circ} 42^{\prime} 12.42^{\prime \prime}$ & $85^{\circ} 18^{\prime} 38.34^{\prime \prime}$ & 1311 & Major vegetable market of the capital city \\
\hline K3 & Industrial area & $27^{\circ} 43^{\prime} 53.69^{\prime \prime}$ & $85^{\circ} 17^{\prime} 54.28^{\prime \prime}$ & 1315 & Industrial area in the north of Kathmandu city \\
\hline K4 & Tourist & $27^{\circ} 42^{\prime} 35.68^{\prime \prime}$ & $85^{\circ} 20^{\prime} 43.87^{\prime \prime}$ & 1318 & $\begin{array}{l}\text { A famous religious place for Hindu people, one of UNESCO-world } \\
\text { heritage site }\end{array}$ \\
\hline K5 & Residential & $27^{\circ} 41^{\prime} 13.45^{\prime \prime}$ & $85^{\circ} 18^{\prime} 8.71 "$ & 1293 & Residential area in Kathmandu \\
\hline K6 & Farm/Industrial & $27^{\circ} 40^{\prime} 12.11^{\prime \prime}$ & $85^{\circ} 25^{\prime} 31.73^{\prime \prime}$ & 1348 & $\begin{array}{l}\text { Eastern part of Kathmandu valley, mix of farm land and industrial } \\
\text { plants }\end{array}$ \\
\hline \multicolumn{6}{|c|}{ Pokhara $\left(28^{\circ} 15^{\prime} N^{\prime} ; 8^{\circ} 58^{\prime} \mathrm{E}\right)$} \\
\hline $\mathrm{P} 1$ & Cropland & $28^{\circ} 16^{\prime} 52.51^{\prime \prime}$ & $83^{\circ} 55^{\prime} 44.17^{\prime \prime}$ & 1065 & Suburban region, famous for maize, paddy production \\
\hline $\mathrm{P} 2$ & $\begin{array}{l}\text { Vegetable } \\
\text { production } \\
\text { area (market) }\end{array}$ & $28^{\circ} 13^{\prime} 39.01^{\prime \prime}$ & $83^{\circ} 58^{\prime} 56.18^{\prime}$ & 871 & $\begin{array}{l}\text { Major market area on one side, vegetable production area on the } \\
\text { other side }\end{array}$ \\
\hline P3 & Industrial area & $28^{\circ} 12^{\prime} 51.39^{\prime \prime}$ & $84^{\circ} 00^{\prime} 37.58^{\prime}$ & 813 & Industrial area for making chocolate and noodles \\
\hline P4 & Tourist place & $28^{\circ} 12^{\prime} 47.35^{\prime \prime}$ & $83^{\circ} 57^{\prime} 41.88^{\prime}$, & 781 & East bank of Phewa lake, a famous tourist destination \\
\hline \multicolumn{6}{|c|}{ Hetauda $\left(27^{\circ} 25^{\prime} \mathrm{N} ; 8^{\circ} 02^{\prime} \mathrm{E}\right)$} \\
\hline H1 & Cropland & $27^{\circ} 23 \prime 26.88^{\prime \prime}$ & $85^{\circ} 02^{\prime} 38.64^{\prime \prime}$ & 512 & A rural village with seasonal farming mostly rice, wheat and mustard \\
\hline $\mathrm{H} 2$ & $\begin{array}{l}\text { Vegetable } \\
\text { production } \\
\text { area }\end{array}$ & $27^{\circ} 25^{\prime} 8.40^{\prime \prime}$ & $85^{\circ} 02^{\prime} 28.20^{\prime \prime}$ & 459 & $\begin{array}{l}\text { Vegetable production area and market, mainly for commercial } \\
\text { purpose }\end{array}$ \\
\hline H3 & Industrial area & $27^{\circ} 24^{\prime} 6.78^{\prime \prime}$ & $85^{\circ} 01 ' 32.34 "$ & 436 & Industrial area for instant food \\
\hline
\end{tabular}




\begin{tabular}{cccccc}
\hline \multicolumn{2}{c}{ Kathmandu and Pokhara (2014-08 to 2015-08) } & \multicolumn{3}{c}{ Hetaunda (2015-11 to 2016-11) } \\
\hline SN & Period & total days & SN & Period & total days \\
\hline 1 & $2014-08-19$ to 2014-10-12 & 55 & 1 & $2015-11-06$ to 2016-01-06 & 62 \\
2 & $2014-10-12$ to 2014-12-11 & 61 & 2 & $2016-01-06$ to 2016-03-05 & 59 \\
3 & $2014-12-11$ to 2015-02-03 & 65 & 3 & $2016-03-05$ to 2016-05-07 & 64 \\
4 & $2015-02-03$ to 2015- 04-18 & 65 & 4 & $2016-05-07$ to 2016-07-09 & 63 \\
5 & $2015-04-18$ to 2015-06-16 & 57 & 5 & $2016-07-09$ to 2016-09-10 & 63 \\
6 & $2015-06-13$ to 2015-08-15 & 64 & 6 & $2016-09-10$ to 2016-11-10 & 62 \\
\hline
\end{tabular}

Table SI-2. PUF-PAS sampling time
68

\section{Text SI-2. Chemical cleanup procedure}

Each extract was concentrated using rotary evaporator and solvent exchanged to hexane. The concentrated extract was loaded on the top of a chromatography column (from the top to bottom: $1 \mathrm{~cm}$ of anhydrous $\mathrm{Na}_{2} \mathrm{SO}_{4}, 2 \mathrm{~g}$ activated alumina, and $3 \mathrm{~g}$ activated silica gel), and eluted with $30 \mathrm{~mL}$ mixture of DCM and hexane (1:1). The volume of eluate was reduced under gentle stream of high purity nitrogen to about $1 \mathrm{ml}$ and added $20 \mu 1$ internal standard containing a known quantity of pentachloronitrobenzene (PCNB) and decachlorobiphenyl (PCB-209). Finally, the volume was reduced to $100 \mu 1$ under gentle stream of nitrogen before analysis.

\section{Text SI-3. Details about the gas chromatography temperature program}

Helium was used as the carrier gas at $1 \mathrm{~mL} \mathrm{~min}^{-1}$ under constant-flow mode. The oven temperature began at $100{ }^{\circ} \mathrm{C}$ for $2 \mathrm{~min}$, ramped up at a rate of $20^{\circ} \mathrm{C} \mathrm{min}^{-1}$ to $140^{\circ} \mathrm{C}$, at $4{ }^{\circ} \mathrm{C} \mathrm{min}^{-1}$ to $200^{\circ} \mathrm{C}(10 \mathrm{~min}$ hold time), then at $4^{\circ} \mathrm{C} \min ^{-1}$ to $310^{\circ} \mathrm{C}$ and held for $5 \mathrm{~min}$. 
Table SI-3. Data of Field blanks and Method detection limits (MDL) ng/PAS

85

\begin{tabular}{|c|c|c|c|c|c|c|c|c|c|c|c|c|c|c|}
\hline \multicolumn{6}{|c|}{ Kathmandu } & \multicolumn{3}{|c|}{ Pokhara } & \multicolumn{3}{|c|}{ Hetauda } & \multicolumn{3}{|c|}{ MDL* } \\
\hline & $\mathrm{Kfb}-1$ & $\mathrm{Kfb}-2$ & $\mathrm{Kfb}-3$ & Kfb-4 & Kfb-5 & Pfb-1 & Pfb-2 & Pfb-3 & Hfb-1 & Hfb-2 & Hfb-3 & $\mathrm{Ktm}$ & $\mathrm{Pkr}$ & Het \\
\hline$o, p^{\prime}-D D T$ & 0.14 & ND & ND & ND & ND & ND & ND & 0.01 & ND & ND & $\mathrm{ND}$ & 0.11 & 0.01 & 0.01 \\
\hline$p, p^{\prime}-D D T$ & 0.02 & ND & ND & ND & ND & ND & ND & 0.06 & 0.02 & 0.01 & 0.01 & 0.02 & 0.06 & 0.02 \\
\hline$o, p^{\prime}-D D E$ & 0.17 & ND & ND & ND & ND & ND & ND & ND & 0.02 & 0.01 & 0.02 & 0.14 & 0.18 & 0.03 \\
\hline$p, p^{\prime}-D D E$ & 1.53 & ND & ND & 0.01 & 0.03 & 0.02 & 0.04 & 0.06 & 0.02 & 0.01 & 0.01 & 1.27 & 0.06 & 0.03 \\
\hline$o, p^{\prime}-D D D$ & ND & ND & ND & ND & ND & ND & ND & ND & ND & ND & ND & 0.22 & 0.22 & 0.01 \\
\hline$p, p^{\prime}-D D D$ & ND & ND & ND & ND & ND & ND & ND & ND & ND & ND & ND & 0.03 & 0.03 & 0.01 \\
\hline$\alpha-H C H$ & 0.05 & ND & ND & 0.01 & 0.02 & 0.02 & 0.01 & ND & 0.02 & 0.07 & ND & 0.04 & 0.02 & 0.13 \\
\hline$\beta-H C H$ & 0.31 & 0.11 & ND & 0.06 & 0.02 & 0.06 & 0.04 & 0.04 & ND & ND & ND & 0.27 & 0.07 & 0.22 \\
\hline$\gamma-H C H$ & 0.19 & ND & ND & 0.01 & 0.06 & 0.03 & 0.01 & 0.01 & ND & 0.01 & 0.02 & 0.16 & 0.03 & 0.01 \\
\hline$\delta-H C H$ & 0.02 & ND & 0 & ND & ND & ND & ND & ND & ND & ND & ND & 0.02 & 0.01 & 0.03 \\
\hline$H C B$ & 0.3 & 0.08 & 0.04 & 0.1 & 0.18 & 0.3 & 0.06 & 0.08 & 0.06 & 0.13 & 0.04 & 0.48 & 0.51 & 0.51 \\
\hline$\alpha$-endo & 0.09 & 0.02 & ND & ND & 0.03 & ND & ND & ND & ND & ND & 0.02 & 0.08 & 0.06 & 0.06 \\
\hline$\beta$-endo & 1.61 & ND & ND & ND & ND & 0.08 & ND & ND & 0.05 & ND & 0.19 & 1.34 & 0.07 & 0.07 \\
\hline Hept & ND & ND & ND & ND & ND & ND & ND & ND & ND & ND & ND & 0.12 & 0.12 & 0.12 \\
\hline Hepx & ND & ND & ND & ND & ND & ND & ND & ND & ND & ND & ND & 0.11 & 0.11 & 0.11 \\
\hline$P C B-28$ & 0.05 & ND & ND & 0.01 & 0.01 & 0.01 & ND & 0.01 & ND & ND & ND & 0.04 & 0.02 & 0.02 \\
\hline$P C B-52$ & ND & ND & ND & ND & ND & ND & ND & ND & ND & ND & ND & 0.08 & 0.08 & 0.08 \\
\hline$P C B-101$ & 0.07 & ND & ND & ND & ND & ND & ND & ND & ND & ND & 0.35 & 0.11 & 0.11 & 0.11 \\
\hline$P C B-153$ & ND & ND & ND & ND & ND & ND & ND & ND & ND & ND & 0.06 & 0.11 & 0.11 & 0.11 \\
\hline$P C B-138$ & ND & ND & ND & ND & ND & ND & ND & ND & ND & ND & ND & 0.08 & 0.08 & 0.08 \\
\hline$P C B-180$ & ND & ND & ND & ND & ND & ND & ND & ND & ND & ND & 0.01 & 0.13 & 0.13 & 0.13 \\
\hline
\end{tabular}

*MDL Method detection limit 
Text SI-4. Details about PUF-disk and Sampling rate

\section{a. Sample holder and sampler}

\section{Dimensions of PUF-Disks:}

Polyurethane foam disk used for air sampling had the following dimensions: Diameter $(\mathrm{d})=135.5$ $\mathrm{cm}$, thickness $(\mathrm{h})=1.3 \mathrm{~cm}$; mass $(\mathrm{m})=3.78 \mathrm{~g}$; area $(\mathrm{A})=341.4 \mathrm{~cm}^{2}\left(\mathrm{ND} 34 \mathrm{~m}^{2}\right)$; Volume $(\mathrm{V})=$ $186.1 \mathrm{~cm}^{3}\left(\mathrm{ND} 00186 \mathrm{~m}^{3}\right)$; Density $(\delta)=20305.6 \mathrm{~g} / \mathrm{m}^{3}$.

A chamber to house the PUF-disk was prepared connecting two stainless steel bowls by means of hinges and a lock. It was so designed that it would protect the PUF-disk from direct precipitation, sunlight and course particle deposition and allow ambient air to pass the through chamber from the gap between bowls and small holes at the base of bottom bowl. This design of chamber has been successfully calibrated and used in numerous previous studies (Shoeib \& Harner, 2002a; Harner et al., 2004; Pozo et al., 2006; Harner et al., 2006 ). PUF disks samplers were pre-cleaned by Soxhlet extraction using dichloromethane (DCM) for $24 \mathrm{~h}$ and dried for $24 \mathrm{~h}$ in a clean desiccator under reduced pressure. Before sending for field deployment, the PUF-disks were spiked with four performance reference compounds (PRCs, PCB-30, -54, -104, -188), that were used to determine the site-specific sampling rates (Pozo et al., 2009). After applying DCs, each PUF-disks was wrapped with clean aluminum foil packed into a plastic bag and stored in a tin container. Five field blanks for Kathmandu, 3 for Pokhara and 3 for Hetauda were prepared to inspect the possible contamination during handling, storage, and transport.

\section{b. Calculation of Sampling Rate " $R$ "}

To assess the site specific sampling rates, PRCs were added to each PUF disks prior to their deployment. Loss of DCs during sampling period was quantified based on individual recoveries. Ideally, recoveries between 20 and $80 \%$ of their initial amount would indicate the linear sampling of individual PAS. This requires DCs with a wide range of octanol-air partition coefficients $\left(\mathrm{K}_{\mathrm{OA}}\right)$. By measuring the loss of DCs during sampling period site-specific air sampling rate ' $R$ ' can be estimated using the following relationship given by Moeckel et al., (2009)

$$
R=\frac{-\ln \left(\frac{C_{D C}^{c o r r}}{C_{D C, 0}}\right) \cdot K_{P A S-A} \cdot \rho_{P A S} \cdot V}{t}
$$

$$
\text { With } C_{D C}^{\text {corr }}=\frac{C_{D C}}{\frac{C_{D C-\text { stable }}}{C_{D C-\text { stable }, 0}}}
$$

$$
K_{P A S-A}=10^{0.6366 \log K_{O A}-3.1774}
$$

Where $C_{D C}$ and $C_{D C \text {-stable }}$ are the concentrations of DC and DC-stable at the end of the deployment period, respectively (ng sample $\left.{ }^{-1}\right) . K_{P A S-A}$ is the chemical's PAS-air partition coefficient with units of $\mathrm{m}^{3} \mathrm{~g}^{-1}$ and it can be calculated according to the regression (eq 2) given by Shoeib and Harner, (2002), $\rho_{P A S}$ is the PAS bulk density $\left(\mathrm{g} \mathrm{m}^{-3}\right), V$ is the volume of the PAS $\left(\mathrm{m}^{3}\right)$, and $t$ is the deployment period in days. PCB-188 is used as DC-stable for correcting the losses of DCs. Only DCs that have recoveries within the desired range of between $20 \%$ and $80 \%$ should be used to estimate uptake rates. 
Table SI-4. Site specific sampling rate

127

\begin{tabular}{cccccccccccccc}
\hline & \multicolumn{2}{c}{ Aug-Oct } & \multicolumn{2}{c}{ Oct-Dec } & \multicolumn{2}{c}{ Dec-Feb } & \multicolumn{2}{c}{ Feb-Apr } & \multicolumn{2}{c}{ Apr-Jun } & \multicolumn{3}{c}{ Jun-Aug } \\
Sampling period & 55 days & \multicolumn{2}{c}{61 days } & \multicolumn{2}{c}{65 days } & 65 days & \multicolumn{2}{c}{57 days } & \multicolumn{2}{c}{64 days } \\
\hline KATHMANDU & I & II & I & II & I & II & I & II & I & II & I & I \\
\hline K1 & 4.4 & 3.9 & 4.6 & 5.2 & 5.2 & 5.3 & 4.5 & 4.7 & 4.3 & 4.4 & 3.3 & 3.4 \\
K2 & 4.3 & 4.4 & 4.9 & 4.6 & 5.2 & 4.6 & 4.7 & 4.3 & 5.5 & NA & 3.4 & 4.1 \\
K3 & 3 & 4.2 & 5.2 & 3.2 & 4 & 3.5 & 3.4 & 3.8 & 4 & 4.1 & 3.1 & 2.3 \\
K4 & 3.9 & 3.7 & 3.4 & 4.2 & 4.2 & NA & 2.9 & 4.4 & 3 & NA & 2.8 & 2.8 \\
K5 & 4.4 & 4.6 & 5 & 3.9 & 5.7 & 6.1 & 3.9 & 4.7 & 4.7 & 4.8 & 3.1 & 3.4 \\
K6 & 4.8 & 4 & 4.3 & 4.3 & 5.9 & 5.4 & 5.4 & 5 & 4.7 & 4.6 & 3.8 & 4.3 \\
\hline Average & $\mathbf{4 . 1}$ & $\mathbf{4 . 1}$ & $\mathbf{4 . 6}$ & $\mathbf{4 . 2}$ & $\mathbf{5}$ & $\mathbf{4 . 9}$ & $\mathbf{4 . 1}$ & $\mathbf{4 . 5}$ & $\mathbf{4 . 4}$ & $\mathbf{4 . 5}$ & $\mathbf{3 . 3}$ & $\mathbf{3 . 4}$ \\
Std. Dev & $\mathbf{0 . 6}$ & $\mathbf{0 . 3}$ & $\mathbf{0 . 7}$ & $\mathbf{0 . 7}$ & $\mathbf{0 . 8}$ & $\mathbf{1}$ & $\mathbf{0 . 9}$ & $\mathbf{0 . 4}$ & $\mathbf{0 . 8}$ & $\mathbf{0 . 3}$ & $\mathbf{0 . 3}$ & $\mathbf{0 . 8}$ \\
\hline POKHARA & & & & & & & & & & & & \\
P1 & 4.7 & 3.7 & 3.6 & 4.1 & 3.9 & 4 & 3.2 & 3.6 & 4.7 & 2.6 & 2.3 & 4.8 \\
P3 & 5.7 & 5.9 & 6.3 & NA & 3.2 & 3.3 & 4.6 & 3.8 & 4.7 & 4.3 & 4 & 4.5 \\
P2 & 6.8 & 5.9 & 3.3 & 3.1 & 2.3 & 2.2 & 2.8 & 2.1 & 5.6 & 5 & 6.1 & 4.9 \\
P4 & 4.8 & 6.4 & 4.9 & 4.8 & 4.4 & 3.7 & 4.2 & 3.5 & 4.9 & 3.7 & 3.9 & 5.7 \\
\hline Average & $\mathbf{5 . 5}$ & $\mathbf{5 . 5}$ & $\mathbf{4 . 5}$ & $\mathbf{4}$ & $\mathbf{3 . 4}$ & $\mathbf{3 . 3}$ & $\mathbf{3 . 7}$ & $\mathbf{3 . 2}$ & $\mathbf{5}$ & $\mathbf{3 . 9}$ & $\mathbf{4 . 1}$ & $\mathbf{5}$ \\
Std. Dev & $\mathbf{1}$ & $\mathbf{1 . 2}$ & $\mathbf{1 . 4}$ & $\mathbf{0 . 8}$ & $\mathbf{0 . 9}$ & $\mathbf{0 . 8}$ & $\mathbf{0 . 8}$ & $\mathbf{0 . 8}$ & $\mathbf{0 . 4}$ & $\mathbf{1}$ & $\mathbf{1 . 6}$ & $\mathbf{0 . 5}$ \\
\hline HETAUDA & 62 & & 62 & & 59 & & 64 & & 64 & & 63 & \\
& days & & days & & days & & days & & days & & days & \\
\hline H1 & NA & NA & 2.1 & 3.9 & 2.7 & 3.2 & 4.7 & 3.7 & 4.8 & 4.9 & 3.2 & 2.8 \\
H2 & NA & NA & 3 & 2.7 & 2.8 & 3.1 & 2.2 & 3 & 3.8 & 3.7 & 3.6 & 4.1 \\
H3 & NA & NA & 2.5 & 2.4 & 3.6 & 4.8 & 2.8 & 2.7 & 3.4 & 2.5 & 2.4 & 4.9 \\
\hline Average & NA & NA & $\mathbf{2 . 5}$ & $\mathbf{3}$ & $\mathbf{3}$ & $\mathbf{3 . 7}$ & $\mathbf{3 . 2}$ & $\mathbf{3 . 1}$ & $\mathbf{4}$ & $\mathbf{3 . 7}$ & $\mathbf{3 . 1}$ & $\mathbf{3 . 9}$ \\
Std. Dev & NA & NA & $\mathbf{0 . 5}$ & $\mathbf{0 . 8}$ & $\mathbf{0 . 5}$ & $\mathbf{0 . 9}$ & $\mathbf{1 . 3}$ & $\mathbf{0 . 5}$ & $\mathbf{0 . 7}$ & $\mathbf{1 . 2}$ & $\mathbf{0 . 6}$ & $\mathbf{1}$ \\
\hline & & & & & & & & & & &
\end{tabular}




\begin{tabular}{|c|c|c|c|c|c|c|c|c|c|c|c|c|c|c|c|c|c|c|}
\hline $\begin{array}{l}\text { Land } \\
\text { type }\end{array}$ & easons & am & & $\begin{array}{l}o, p^{\prime} \\
D D T\end{array}$ & $\begin{array}{l}p, p^{\prime}- \\
D D T\end{array}$ & $\begin{array}{l}o, p^{\prime}- \\
D D E\end{array}$ & $\begin{array}{l}p, p^{\prime}- \\
D D E\end{array}$ & $\begin{array}{l}o, p^{\prime}- \\
D D D \\
\end{array}$ & $\begin{array}{l}p, p^{\prime}- \\
D D D\end{array}$ & $\begin{array}{c}\alpha- \\
H C H \\
\end{array}$ & $\begin{array}{c}\beta- \\
H C H \\
H C\end{array}$ & $\begin{array}{c}\gamma- \\
H C H \\
\end{array}$ & $\begin{array}{c}\delta- \\
H C H \\
\end{array}$ & $H C B$ & $\begin{array}{c}\alpha- \\
\text { endo } \\
\end{array}$ & $\begin{array}{c}\beta- \\
\text { endo }\end{array}$ & Hept & Нерx \\
\hline \multirow{12}{*}{$\begin{array}{l}\overrightarrow{\widetilde{J}} \\
\text { త్ } \\
\stackrel{0}{0} \\
\stackrel{0}{0}\end{array}$} & \multirow{4}{*}{ Winter } & & I & 8.1 & 4.9 & 2 & 8.4 & 0.6 & 0.7 & 6.8 & 2.5 & 7.6 & 1.1 & 20.2 & 3.3 & 3.4 & BDL & BDL \\
\hline & & & II & 7.9 & 5.3 & 1.7 & 5.8 & 0.4 & 0.8 & 6 & 2.4 & 6.7 & 2.7 & 25.8 & 1.7 & 10 & 0.1 & BDL \\
\hline & & \multirow{2}{*}{2} & I & 5.5 & 3.1 & 0.9 & 4.6 & BDL & 0.9 & 5.3 & 0.3 & 6.7 & 2.1 & 23.9 & BDL & 2.5 & BDL & 3DL \\
\hline & & & II & 4.4 & 2.8 & 0.6 & 3.9 & BDL & 0.3 & 3.1 & 0.8 & 3.7 & 1 & 23 & BDL & 2.5 & BDL & BDL \\
\hline & \multirow{4}{*}{$\begin{array}{c}\text { Pre- } \\
\text { monsoon }\end{array}$} & \multirow{2}{*}{3} & I & 26.1 & 25.9 & 3.4 & 13.8 & BDL & 1.1 & 7.4 & 2 & 17.8 & BDL & 15.4 & 1 & 6.9 & BDL & BDL \\
\hline & & & II & 23.4 & 16.9 & 2.9 & 8.9 & BDL & 0.8 & 4.5 & 1.2 & 12.8 & BDL & 15.8 & BDL & 1.3 & BDL & BDL \\
\hline & & & I & 61.9 & 72.2 & 3.1 & 25.6 & 1.1 & 2.4 & 10.9 & 2.7 & 17.3 & 2.8 & 21.2 & 3.2 & 8.6 & BDL & BDL \\
\hline & & & II & 56 & 66.4 & 4.3 & 21.1 & 1.1 & 2.3 & 9.1 & 1.4 & 14.1 & 1.3 & 22.7 & BDL & BDL & BDL & BDL \\
\hline & \multirow{4}{*}{ Monsoon } & & I & 40.3 & 40.8 & 2.3 & 13.3 & 0.8 & 1.2 & 12.3 & 1.6 & 12.3 & 1 & 16.1 & 1.5 & 7.2 & BDL & BDL \\
\hline & & & II & 48 & 49.3 & 3 & 24.1 & 1.3 & 1.9 & 18.2 & 1.7 & 14.9 & 0.9 & 28.3 & BDL & BDL & BDL & 3DL \\
\hline & & 6 & I & 24.4 & 19.5 & 2.9 & 15.2 & 0.5 & 1.3 & 12.3 & 2 & 12.8 & 3 & 31.3 & 2.4 & 5.1 & BDL & BDL \\
\hline & & 0 & II & 28.3 & 24 & 2.2 & 15.4 & BDL & 0.8 & 10.1 & 2.7 & 7.1 & 1.5 & 23.1 & BDL & 10.5 & BDL & BDL \\
\hline \multirow{11}{*}{ 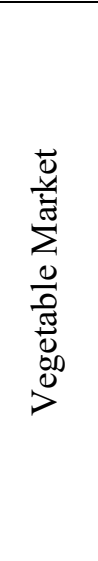 } & \multirow{4}{*}{ Winter } & & I & 11.9 & 20.1 & 1.8 & 26.8 & 0.7 & 3.2 & 14.2 & 2.4 & 26.6 & 2.4 & 20.5 & BDL & 7.7 & BDL & $\overline{B D L}$ \\
\hline & & & II & 13.4 & 22 & 1.4 & 29.4 & 0.7 & 3.5 & 10.5 & 2.4 & 27.1 & 0.6 & 31.7 & BDL & 8.3 & 0.4 & BDL \\
\hline & & & I & 6.5 & 14.9 & 1.4 & 25.5 & 2. & 4.2 & 6.1 & 2.9 & 18.8 & 0.4 & 45.5 & 2 & 13.2 & 0.9 & BDL \\
\hline & & & II & 6.4 & 11.6 & 1.7 & 26 & 1. & 4.1 & 7.6 & 1.6 & 19.3 & BDL & 38.7 & BDL & 4.3 & 0.8 & BDL \\
\hline & \multirow{3}{*}{$\begin{array}{c}\text { Pre- } \\
\text { monsoon }\end{array}$} & & I & 48.4 & 102.9 & 9.6 & 112.1 & 3.7 & 10.5 & 15.6 & 4 & 63.4 & 1.4 & 27.9 & BDL & 12.6 & 0.5 & BDL \\
\hline & & & II & 52.6 & 130.7 & 8 & 125 & 4.5 & 13.4 & 18.1 & 7.6 & 62.2 & 2.6 & 25.9 & 7.3 & 21.7 & 0.9 & BDL \\
\hline & & 4 & I & 50.1 & 96.9 & 6.5 & 65.8 & 2.6 & 6.1 & 9.3 & 3.4 & 44.5 & 2.6 & 16.3 & 7.1 & 3.8 & 0.9 & BDL \\
\hline & \multirow{4}{*}{ Monsoc } & & I & 89.5 & 144.8 & 11.2 & 186.7 & 4.9 & 9.8 & 28.2 & 6.6 & 229.2 & 5 & 28.3 & 15.7 & 35.3 & 0.7 & BDL \\
\hline & & & II & 75.5 & 111.1 & 12.3 & 145.7 & 3.9 & 7.3 & 21.4 & 7.7 & 182.2 & 6.4 & 28.2 & 8 & 4.9 & 1 & BDL \\
\hline & & & I & 48.9 & 104.6 & 3.9 & 92.6 & 2. & 6.2 & 19.1 & 4.3 & 30.8 & 7.4 & 31.3 & 4.3 & 18.2 & 0.2 & BDL \\
\hline & & 0 & II & 49.9 & 104.9 & 5.6 & 97.6 & 3. & 6.7 & 22.9 & 4.8 & 37.9 & 9.6 & 25.5 & BDL & 17.8 & 0.4 & BDL \\
\hline \multirow{6}{*}{ 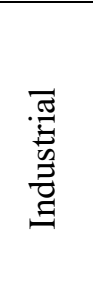 } & \multirow{4}{*}{ Winter } & & I & 5.4 & 5.9 & 1.7 & 11.6 & 0. & 1.9 & 6.3 & 1.4 & 9.7 & BDL & 135.1 & BDL & 2.4 & 0.7 & $\overline{B D L}$ \\
\hline & & & II & 3.6 & 3.8 & 0.7 & 6.5 & 0.2 & 0.8 & 4.2 & 1.2 & 6 & BDL & 73.7 & BDL & 1.1 & 0.4 & BDL \\
\hline & & & I & 6.3 & 22.9 & 1.6 & 14.6 & 0.5 & 3.7 & 7.1 & 3.1 & 15 & 2.9 & 242.5 & 1.7 & 3.3 & 0.2 & BDL \\
\hline & & & II & 6.2 & 23.1 & 1.9 & 16.6 & 0.5 & 4.2 & 7. & 1.3 & 20.9 & BDL & 347 & 3.8 & 2.9 & 0.3 & BDL \\
\hline & \multirow{2}{*}{$\begin{array}{c}\text { Pre- } \\
\text { monsoon }\end{array}$} & \multirow{2}{*}{3} & I & 18 & 19.9 & 2 & 18.7 & 0.7 & 2.7 & 8.5 & 2.3 & 16.8 & 1.4 & 113.1 & 2.4 & 4.3 & 0.3 & BDL \\
\hline & & & II & 15 & 15 & 2.6 & 16.8 & 0.5 & 2.3 & 8 & 1.6 & 20.7 & 1.5 & 104.9 & 2.4 & 6.3 & BDL & BDL \\
\hline
\end{tabular}




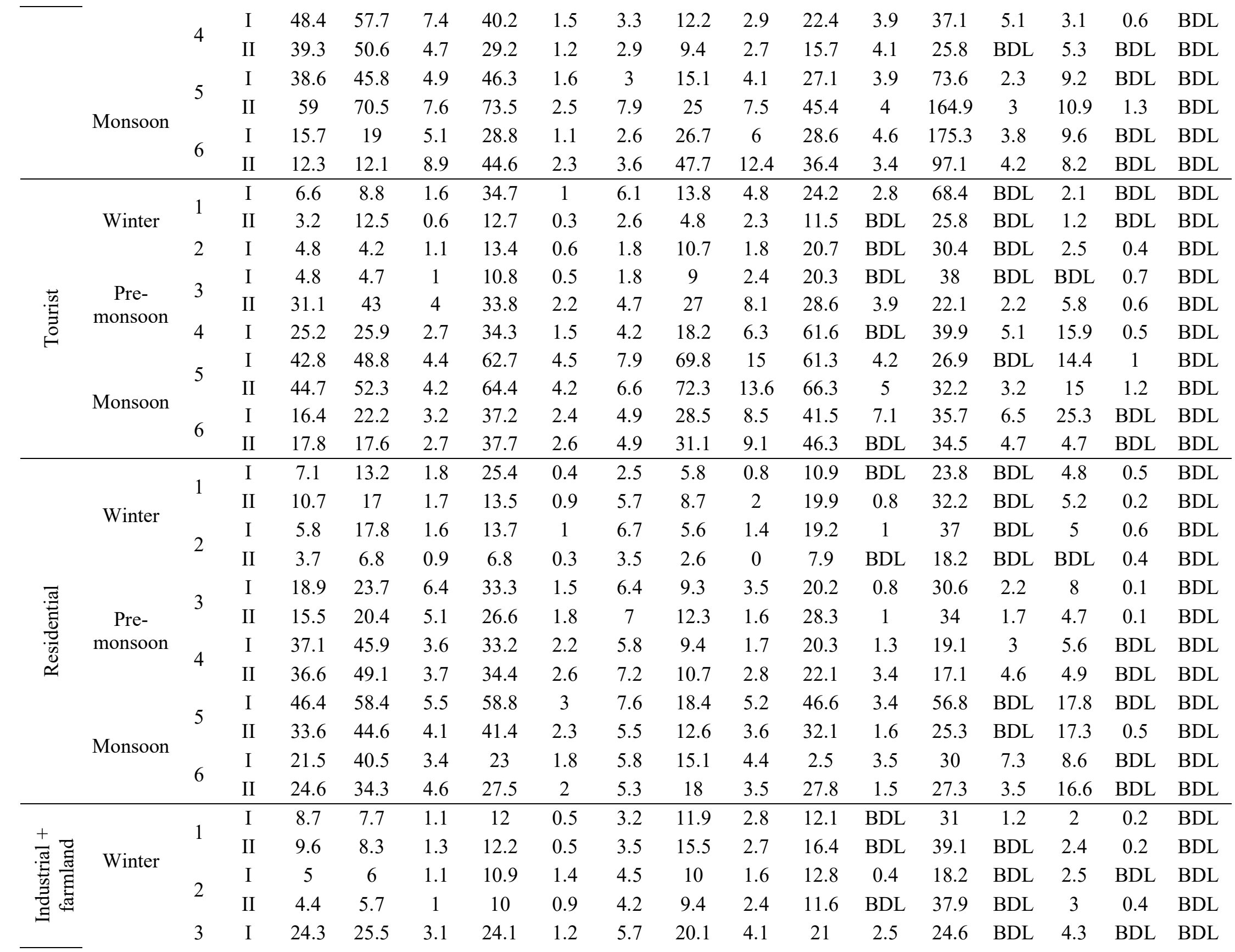




\begin{tabular}{|c|c|c|c|c|c|c|c|c|c|c|c|c|c|c|c|c|c|}
\hline \multirow{3}{*}{$\begin{array}{c}\text { Pre- } \\
\text { monsoon }\end{array}$} & & II & 25.7 & 25.4 & 3.6 & 26.2 & 1.2 & 6.3 & 13.4 & 4.2 & 20 & BDL & 23.1 & 2.3 & 6.2 & BDL & BDL \\
\hline & \multirow{2}{*}{4} & I & 32.5 & 43.3 & 2.1 & 27.2 & 1.5 & 4 & 26.5 & 8.1 & 21.8 & 4.8 & 29.4 & 7.4 & 18.7 & BDL & BDL \\
\hline & & II & 45.7 & 61.3 & 3.3 & 32.6 & 2 & 5.5 & 25.8 & 7.1 & 17.4 & 3.6 & 36.9 & 1.7 & 14.4 & BDL & BDL \\
\hline \multirow{4}{*}{ Monsoon } & \multirow{2}{*}{5} & I & 46.2 & 58.5 & 5 & 48.9 & 3.7 & 10.4 & 62.4 & 14.1 & 38.3 & 4.4 & 87.3 & BDL & 25 & 0.5 & BDL \\
\hline & & II & 52.7 & 71.1 & 5.7 & 54.7 & 3.6 & 10.8 & 72.9 & 16 & 43.9 & 4.5 & 58 & 7.1 & 7.5 & 0.8 & BDL \\
\hline & \multirow{2}{*}{6} & I & 16.1 & 15.8 & 2.3 & 21.4 & 1.3 & 4.4 & 20.5 & 6.5 & 16.6 & 4.4 & 34.8 & 3.4 & 17 & 0.2 & BDL \\
\hline & & II & 22.2 & 20.4 & 2.7 & 28 & 2 & 6 & 35.9 & 10.1 & 19.2 & 5.6 & 48 & BDL & 12.3 & 0.3 & BDL \\
\hline
\end{tabular}

130

\section{Table SI-5b. Site specific concentrations $\left(\mathrm{pg} / \mathrm{m}^{3}\right)$ of OCPs in different urban sites of Pokhara}

\begin{tabular}{|c|c|c|c|c|c|c|c|c|c|c|c|c|c|c|c|c|c|c|}
\hline $\begin{array}{l}\text { Site } \\
\text { type }\end{array}$ & Seasons & & & $\begin{array}{l}o, p^{\prime}- \\
D D T\end{array}$ & $\begin{array}{l}p, p^{\prime}- \\
D D T\end{array}$ & $\begin{array}{l}o, p^{\prime}- \\
D D E\end{array}$ & $\begin{array}{l}p, p^{\prime}- \\
D D E\end{array}$ & $\begin{array}{c}o, p^{\prime}- \\
D D D \\
\end{array}$ & $\begin{array}{l}p, p^{\prime}- \\
D D D\end{array}$ & $\begin{array}{c}\alpha- \\
H C H \\
\end{array}$ & $\begin{array}{c}\beta- \\
H C H \\
\end{array}$ & $\begin{array}{c}\gamma- \\
H C H \\
\end{array}$ & $\begin{array}{c}\delta- \\
H C H \\
\end{array}$ & $H C B$ & $\begin{array}{c}\alpha- \\
\text { endo }\end{array}$ & $\begin{array}{c}\beta- \\
\text { endo }\end{array}$ & Hept & Нерх \\
\hline \multirow{11}{*}{$\begin{array}{l}\overrightarrow{0} \\
\frac{\vec{\sigma}}{2} \\
\stackrel{0}{0} \\
\dot{U}\end{array}$} & \multirow{3}{*}{ Winter } & \multirow{2}{*}{1} & I & 9.5 & 25.6 & 2.8 & 37.9 & BDL & 1.6 & 5.4 & $\overline{B D L}$ & 8 & BDL & 15.1 & $\overline{B D L}$ & 4.7 & 0.5 & BDL \\
\hline & & & II & 6.5 & 17.1 & 1.7 & 24.6 & BDL & 1.1 & 3.3 & 0.4 & 7.2 & 0.3 & 14.2 & 1.2 & 1.4 & BDL & BDL \\
\hline & & 2 & I & 3.4 & 9.6 & 1 & 14.5 & BDL & 0.9 & 3.7 & 2.5 & 15.5 & 0.5 & 19.5 & 1.5 & 1.7 & 0.4 & BDL \\
\hline & \multirow{4}{*}{$\begin{array}{c}\text { Pre- } \\
\text { monsoon }\end{array}$} & \multirow{2}{*}{3} & I & 18.6 & 114.3 & 5.2 & 207.4 & 0.8 & 5.6 & 3.9 & 0.9 & 15.9 & 1.6 & 23.9 & BDL & 5 & 0.7 & BDL \\
\hline & & & II & 13.5 & 95.7 & 4.3 & 182.3 & 0.6 & 5 & 3.9 & 0.7 & 17.7 & $\mathrm{BDL}$ & 21.9 & 0.7 & 2 & 0.2 & BDL \\
\hline & & \multirow{2}{*}{4} & I & 50.1 & 132.6 & 4.7 & 136.8 & 0.9 & 4.5 & 6 & 0.8 & 23.6 & 0.8 & 19.9 & 6.8 & 4.6 & BDL & BDL \\
\hline & & & II & 58.4 & 142.7 & 3.4 & 121.7 & 0.8 & 3.6 & 6.6 & 0.5 & 18.8 & 1.7 & 12.9 & 2.6 & 1.3 & 0.3 & BDL \\
\hline & \multirow{4}{*}{ Monsoon } & \multirow{2}{*}{5} & I & 61.8 & 147.8 & 5.5 & 140.3 & 1.2 & 4.5 & 7.5 & 3.8 & 15.5 & 8.8 & 46.3 & 5.5 & 10.7 & 0.2 & BDL \\
\hline & & & II & 43.1 & 100.9 & 4.4 & 106.6 & BDL & 3.6 & 9 & 3.5 & 15.3 & 3.3 & 34.2 & 1.2 & 3.1 & 0.2 & BDL \\
\hline & & 6 & I & 18.5 & 35.4 & 1.9 & 38.3 & BDL & 1.5 & 6.7 & 1.2 & 6.4 & 1 & 15.4 & 0.7 & 1.2 & BDL & BDL \\
\hline & & 0 & II & 14.6 & 26.2 & 1.4 & 19.8 & BDL & 0.8 & 5.7 & 0.5 & 5.1 & BDL & 13.8 & BDL & 1.1 & 0.2 & BDL \\
\hline \multirow{10}{*}{ 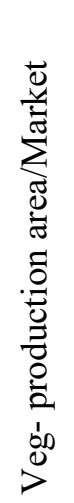 } & \multirow{4}{*}{ Winter } & \multirow{2}{*}{1} & I & 13.9 & 30.3 & 2.2 & 45.3 & 0.6 & 4.4 & 6.3 & 1.5 & 36 & 1.3 & 14.9 & BDL & 3.8 & BDL & $\overline{B D L}$ \\
\hline & & & II & 13 & 25.9 & 1.7 & 34 & 0.7 & 5.1 & 4.9 & BDL & 28.6 & BDL & 22.3 & BDL & 6.2 & 0.2 & BDL \\
\hline & & \multirow{2}{*}{2} & I & 10.4 & 33.3 & 2.1 & 34.3 & 0.5 & 4.8 & 4.5 & BDL & 21.8 & BDL & 12.7 & BDL & 11 & BDL & BDL \\
\hline & & & II & 13.7 & 37 & 2.4 & 40.5 & 0.6 & 6.4 & 5.9 & BDL & 27.2 & BDL & 16.1 & BDL & 4.7 & BDL & BDL \\
\hline & \multirow{3}{*}{$\begin{array}{c}\text { Pre- } \\
\text { monsoon }\end{array}$} & \multirow{2}{*}{3} & I & 27.8 & 47.8 & 3.1 & 51.3 & 0.9 & 4.7 & 4.3 & 1.3 & 26.4 & BDL & 20.7 & BDL & 6.7 & BDL & BDL \\
\hline & & & II & 13.8 & 22 & 2.3 & 31 & 0.6 & 3.1 & 5.1 & 0.6 & 14.7 & 1.1 & 21.3 & 2.1 & 4.2 & BDL & BDL \\
\hline & & 4 & I & 69.1 & 129.2 & 5.5 & 77.1 & 1.4 & 5.7 & 7.2 & 2.1 & 32.7 & 2.5 & 41.6 & BDL & 8.3 & BDL & BDL \\
\hline & \multirow{3}{*}{ Monsoon } & \multirow[b]{3}{*}{6} & I & 21.7 & 620.7 & 11.6 & 364.1 & 2.2 & BDL & 5.8 & 2.3 & 30.5 & 2.7 & 21.8 & BDL & 4.4 & $\mathrm{BDL}$ & BDL \\
\hline & & & II & 20.3 & 530.4 & 9.2 & 291.9 & 1.9 & 19.7 & 5.5 & 1.4 & 20.8 & 3.6 & 17.5 & BDL & 3.1 & 0.1 & BDL \\
\hline & & & I & 43.3 & 79 & 4 & 69.1 & 1.2 & 3.4 & 10.6 & 1.7 & 25.6 & BDL & 14.2 & 2.8 & 2.2 & BDL & BDL \\
\hline
\end{tabular}




\begin{tabular}{|c|c|c|c|c|c|c|c|c|c|c|c|c|c|c|c|c|c|c|}
\hline & & & II & 27.4 & 48.6 & 2.4 & 43.2 & 0.8 & 2.9 & 6.4 & 1.4 & 15.8 & 0.7 & 7.3 & 3.5 & 5.9 & BDL & $\mathrm{BDL}$ \\
\hline \multirow{12}{*}{ 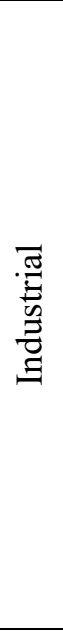 } & \multirow{4}{*}{ Winter } & \multirow{2}{*}{1} & I & 15.4 & 24 & 3 & 34.3 & 0.8 & 2.8 & 5.6 & 1.6 & 10.6 & 1.2 & 25.6 & 0.4 & 3.9 & BDL & BDL \\
\hline & & & II & 15.2 & 17.8 & 1.9 & 24.1 & 0.5 & 2.6 & 6.6 & BDL & 11 & BDL & 20.2 & 3.3 & 2.4 & 0.4 & BDL \\
\hline & & \multirow{2}{*}{2} & I & 12.9 & 25.9 & 3.8 & 45.3 & 1.2 & 5.6 & 5.4 & 1.9 & 17.4 & 1.5 & 42.9 & 6.7 & 3.9 & BDL & BDL \\
\hline & & & II & 18.9 & 20.9 & 3 & 26.6 & 2.4 & 9.7 & 11.5 & 5.5 & 38.8 & 1.8 & 35.1 & BDL & 6.5 & BDL & BDL \\
\hline & \multirow{4}{*}{$\begin{array}{c}\text { Pre- } \\
\text { monsoon }\end{array}$} & \multirow{2}{*}{3} & I & 9.1 & 46.9 & 2.1 & 21 & 0 & 1.7 & 6.4 & 1 & 21 & 1.5 & 18.8 & 8 & 3.9 & BDL & BDL \\
\hline & & & II & 14.5 & 6.2 & 3.3 & 31.7 & 0.1 & 2.7 & 7.8 & 1.4 & 24.8 & 2 & 22.2 & 12.6 & 6.2 & BDL & BDL \\
\hline & & \multirow{2}{*}{4} & I & 51.9 & 77.5 & 3.4 & 40.7 & 1.5 & 3.8 & 7.4 & 0.7 & 13 & 1.8 & 23.2 & BDL & 4.3 & BDL & BDL \\
\hline & & & II & 44.8 & 65.7 & 3 & 33.9 & 1 & 2.7 & 5.4 & 0.3 & 9.1 & 2.1 & 18.6 & 1.8 & 11.5 & BDL & BDL \\
\hline & \multirow{4}{*}{ Monsoon } & \multirow{2}{*}{5} & I & 27.2 & 37.2 & 2.7 & 28.7 & 1 & 2.6 & 3.5 & 0.7 & 6.8 & 0.5 & 17.3 & BDL & 4.1 & BDL & BDL \\
\hline & & & II & 33.7 & 43.6 & 2.2 & 29.4 & 1 & 2.7 & 3.3 & 0.4 & 5 & 0.4 & 11.5 & BDL & 4.6 & BDL & BDL \\
\hline & & \multirow{2}{*}{6} & I & 21.9 & 27.2 & 2 & 24.7 & 1.3 & 3.2 & 11.5 & 1.1 & 14.5 & 2.9 & 23.7 & 2.8 & 3.4 & BDL & BDL \\
\hline & & & II & 24.2 & 35.4 & 3.2 & 35.2 & 0.9 & 2 & 9.5 & 2.6 & 12.6 & 2.8 & 24.5 & 6.9 & 4.2 & BDL & BDL \\
\hline \multirow{11}{*}{ 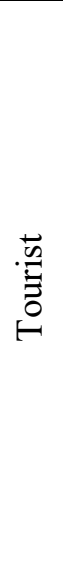 } & \multirow{3}{*}{ Winter } & 1 & I & 4.7 & 6.3 & 0.7 & 8.8 & 0.4 & 1.7 & 3.1 & BDL & 6.9 & 1.6 & 12.8 & 2.4 & 6.5 & BDL & BDL \\
\hline & & \multirow{2}{*}{2} & I & 7.1 & 22.6 & 1.5 & 29.1 & BDL & 2.2 & 2.8 & BDL & 18.9 & BDL & 11.6 & BDL & 6 & BDL & BDL \\
\hline & & & II & 7.7 & 23.1 & 1.4 & 21.3 & 0.3 & 1.9 & 3.2 & 0.4 & 17.7 & BDL & 15.4 & BDL & 10.9 & BDL & BDL \\
\hline & \multirow{4}{*}{$\begin{array}{c}\text { Pre- } \\
\text { monsoon }\end{array}$} & \multirow{2}{*}{3} & I & 19.7 & 27.4 & 2.9 & 28.4 & 0.8 & 2.1 & 3.4 & 0.9 & 13 & 1.2 & 31.7 & BDL & 7.5 & BDL & BDL \\
\hline & & & II & 15.8 & 17.9 & 1.7 & 18.8 & 1.2 & 3 & 3.7 & 2 & 11.3 & 0.8 & 13.5 & BDL & 2.5 & BDL & BDL \\
\hline & & \multirow{2}{*}{4} & I & 49.1 & 71.4 & 4.4 & 39.7 & 1.9 & 4.1 & 4.5 & 1 & 14.6 & 1.5 & 21.4 & BDL & 6 & BDL & BDL \\
\hline & & & II & 29.5 & 39.9 & 4.1 & 25.6 & 1.7 & 3 & 2.6 & 0.6 & 7.4 & 1.4 & 6.9 & 2.2 & 3.1 & BDL & BDL \\
\hline & \multirow{4}{*}{ Monsoon } & \multirow{2}{*}{5} & I & 7.6 & 23 & 1.3 & 17.8 & 0.5 & 1.2 & 3.3 & 1.7 & 32.3 & 3.3 & 18.5 & 2.3 & 4 & BDL & BDL \\
\hline & & & II & 5.3 & 18.3 & 1.1 & 13.6 & 0.4 & 0.8 & 3.2 & 1.9 & 34.8 & 4.1 & 14.6 & 2 & 1.9 & 0.3 & BDL \\
\hline & & \multirow{2}{*}{6} & I & 16.6 & 24.1 & 2.1 & 26.5 & 0.9 & 2.1 & 7.3 & 1.4 & 10.8 & 0.8 & 18.9 & BDL & 2.7 & BDL & BDL \\
\hline & & & II & 20 & 31.8 & 5 & 52 & 1.8 & 3.6 & 14.1 & 2.7 & 23.2 & 5.2 & 15.6 & 3.2 & 7.3 & BDL & BDL \\
\hline
\end{tabular}

132

133

134 


\begin{tabular}{|c|c|c|c|c|c|c|c|c|c|c|c|c|c|c|c|c|c|c|}
\hline Site type & Seasons & & ling & $\begin{array}{l}o, p^{\prime}- \\
D D T\end{array}$ & $\begin{array}{l}p, p^{\prime}- \\
D D T\end{array}$ & $\begin{array}{l}o, p^{\prime}- \\
D D E\end{array}$ & $\begin{array}{l}p, p^{\prime}- \\
D D E\end{array}$ & $\begin{array}{l}o, p^{\prime}- \\
D D D\end{array}$ & $\begin{array}{l}p, p^{\prime}- \\
D D D\end{array}$ & $\begin{array}{c}\alpha- \\
H C H \\
\end{array}$ & $\begin{array}{c}\beta- \\
H C H \\
\end{array}$ & $\begin{array}{c}\gamma- \\
H C H \\
\end{array}$ & $\begin{array}{c}\delta- \\
H C H\end{array}$ & $H C B$ & $\begin{array}{c}\alpha- \\
\text { endo } \\
\end{array}$ & $\begin{array}{c}\beta- \\
\text { endo } \\
\end{array}$ & Hept & Hepx \\
\hline \multirow{10}{*}{ 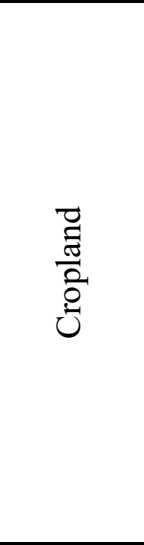 } & \multirow{4}{*}{ Winter } & \multirow{2}{*}{1} & I & 1.3 & 8.9 & 0.3 & 3.4 & BDL & 0.6 & 6.2 & 1.2 & 10.5 & 0.9 & 23.2 & 1.4 & 7.7 & BDL & BDL \\
\hline & & & II & 1.0 & 5.0 & 0.3 & 2.2 & BDL & 0.1 & 6.0 & 0.9 & 6.0 & 0.8 & 27.6 & 1.1 & 4.8 & BDL & BDL \\
\hline & & \multirow{2}{*}{2} & I & 1.7 & 8.1 & 0.3 & 3.3 & 0.1 & 0.2 & 6.5 & 1.5 & 12.5 & 2.9 & 17.0 & 1.5 & 10.1 & BDL & BDL \\
\hline & & & II & 2.4 & 9.9 & 0.3 & 3.1 & 0.1 & 0.2 & 8.8 & 1.3 & 13.5 & 4.3 & 16.8 & 1.2 & 6.9 & BDL & BDL \\
\hline & \multirow{4}{*}{$\begin{array}{c}\text { Pre- } \\
\text { monsoon }\end{array}$} & \multirow{2}{*}{3} & I & 2.6 & 12.1 & 0.3 & 2.3 & 0.1 & 0.2 & 5.9 & 0.9 & 9.0 & 1.9 & 13.8 & 0.9 & 6.1 & BDL & BDL \\
\hline & & & II & 4.6 & 22.4 & 0.4 & 3.3 & 0.1 & 0.3 & 4.7 & 1.3 & 8.4 & 1.2 & 12.8 & 1.2 & 9.7 & BDL & BDL \\
\hline & & \multirow{2}{*}{4} & I & 3.0 & 11.9 & 0.7 & 10.2 & 0.1 & 0.2 & 3.2 & 2.4 & 4.4 & 1.8 & 7.9 & 3.4 & 27.5 & BDL & BDL \\
\hline & & & II & 4.0 & 11.8 & 0.4 & 3.0 & 0.1 & 0.2 & 2.8 & 0.9 & 4.2 & 0.7 & 8.8 & 0.7 & 3.4 & BDL & BDL \\
\hline & \multirow{2}{*}{ Monsoon } & \multirow{2}{*}{5} & I & 4.5 & 12.8 & 0.3 & 3.6 & 0.1 & 0.2 & 3.4 & 1.2 & 5.7 & 0.6 & 11.5 & 1.3 & 8.8 & BDL & BDL \\
\hline & & & II & 4.5 & 12.1 & 0.5 & 3.9 & 0.1 & 0.2 & 2.7 & 1.5 & 5.4 & 1.0 & 10.9 & 1.0 & 7.4 & BDL & BDL \\
\hline \multirow{10}{*}{ 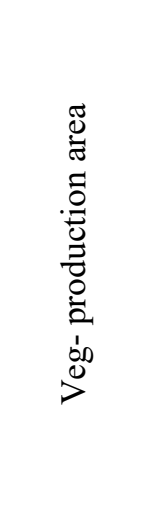 } & \multirow{4}{*}{ Winter } & \multirow{2}{*}{1} & I & 3.1 & 27.4 & 0.6 & 7.4 & 0.2 & 0.4 & 10.2 & BDL & 2960.3 & 4.7 & 16.0 & 1.7 & 14.1 & 1 & BDL \\
\hline & & & II & 1.3 & 5.7 & 0.3 & 4.4 & BDL & 0.2 & 5.3 & BDL & 40.6 & 2.4 & 28.1 & 1.0 & 5.1 & 0.8 & BDL \\
\hline & & \multirow{2}{*}{2} & I & 1.0 & 7.8 & 0.6 & 10.2 & 0.3 & 0.2 & 6.8 & 2.0 & 3192.6 & BDL & 22.3 & 1.9 & 5.6 & BDL & BDL \\
\hline & & & II & 1.2 & 7.8 & 0.5 & 6.7 & 0.1 & 0.3 & 6.6 & BDL & 3326.3 & 1.7 & 20.5 & 0.8 & 3.9 & BDL & BDL \\
\hline & \multirow{4}{*}{$\begin{array}{c}\text { Pre- } \\
\text { monsoon }\end{array}$} & \multirow{2}{*}{3} & I & 3.1 & 29.2 & 0.6 & 10.8 & 0.2 & 0.6 & 9.3 & BDL & 3097.6 & BDL & 20.8 & 3.5 & 15.8 & BDL & BDL \\
\hline & & & II & 2.3 & 12.1 & 0.3 & 7.9 & 0.3 & 0.9 & 6.7 & BDL & 1662.0 & BDL & 16.5 & 2.6 & 11.4 & BDL & BDL \\
\hline & & \multirow{2}{*}{4} & I & 1.8 & 2.4 & 0.1 & 6.5 & 0.5 & 1.1 & 6.1 & BDL & 867.7 & BDL & 16.8 & 2.2 & 9.3 & BDL & BDL \\
\hline & & & II & 0.9 & 7.1 & 0.3 & 3.8 & 0.0 & 0.3 & 1.2 & BDL & 227.0 & 0.6 & 7.9 & 0.9 & 3.0 & BDL & BDL \\
\hline & onson & 5 & I & 2.8 & 16.3 & 0.6 & 7.1 & 0.2 & 0.4 & 4.3 & BDL & 543.6 & 4.5 & 12.5 & 2.4 & 12.3 & 0.8 & BDL \\
\hline & IVIOnsoon & 3 & II & 1.7 & 13.3 & 0.5 & 6.7 & 0.1 & 0.3 & 3.9 & 1.4 & 427.0 & 1.5 & 13.9 & 1.4 & 7.4 & BDL & BDL \\
\hline \multirow{10}{*}{ 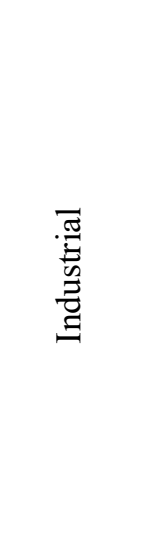 } & \multirow{4}{*}{ Winter } & \multirow{2}{*}{1} & I & 3.1 & 28.1 & 1.1 & 21.8 & 0.3 & 0.1 & 10.8 & 6.8 & 21.2 & 8.4 & 46.8 & 5.5 & 35.6 & BDL & BDL \\
\hline & & & II & 4.1 & 21.5 & 1.1 & 15.7 & 0.2 & 0.6 & 4.8 & 2.4 & 13.9 & 3.5 & 27.2 & 4.6 & 28.6 & BDL & BDL \\
\hline & & \multirow{2}{*}{2} & I & 1.3 & 6.3 & 0.4 & 4.7 & BDL & 0.1 & 1.5 & BDL & 3.1 & 0.6 & 12.6 & 0.8 & 5.6 & BDL & BDL \\
\hline & & & II & 1.0 & 5.5 & 0.3 & 4.0 & BDL & 0.1 & 1.3 & 0.7 & 2.7 & 0.5 & 11.2 & 0.8 & 3.8 & BDL & BDL \\
\hline & \multirow{4}{*}{$\begin{array}{c}\text { Pre- } \\
\text { monsoon }\end{array}$} & \multirow{2}{*}{3} & I & 4.7 & 26.0 & 0.9 & 14.6 & 0.2 & 0.4 & 6.5 & 4.4 & 10.9 & 2.7 & 24.7 & 3.4 & 25.1 & BDL & BDL \\
\hline & & & II & 4.7 & 17.0 & 0.8 & 10.3 & 0.1 & 0.3 & 3.0 & 1.9 & 5.4 & 1.8 & 13.0 & 1.8 & 10.9 & BDL & BDL \\
\hline & & \multirow{2}{*}{4} & $\mathrm{I}$ & 3.4 & 12.5 & 0.7 & 10.4 & 0.1 & 0.2 & 2.6 & 1.5 & 4.7 & 2.5 & 6.8 & 2.8 & 26.6 & BDL & BDL \\
\hline & & & II & 3.3 & 18.1 & 0.8 & 9.7 & 0.2 & 0.1 & 5.8 & 1.8 & 4.0 & 2.2 & 26.5 & 2.5 & 10.5 & BDL & BDL \\
\hline & \multirow{2}{*}{ Monsoon } & 5 & I & 7.1 & 48.9 & 0.9 & 11.6 & 0.4 & 0.1 & 7.2 & 1.3 & 4.1 & 1.6 & 6.2 & 2.4 & 13.5 & $\mathrm{BDL}$ & BDL \\
\hline & & J & II & 4.4 & 29.8 & 0.6 & 7.1 & 0.2 & BDL & 6.2 & 0.8 & 6.0 & 1.0 & 5.8 & 1.6 & 8.4 & BDL & BDL \\
\hline
\end{tabular}




\begin{tabular}{|c|c|c|c|c|c|c|c|c|c|}
\hline $\begin{array}{l}\text { Land } \\
\text { type }\end{array}$ & Seasons & & ling & $\begin{array}{c}P C B- \\
28 \\
\end{array}$ & $\begin{array}{c}P C B- \\
52 \\
\end{array}$ & $\begin{array}{c}P C B- \\
101 \\
\end{array}$ & $\begin{array}{c}P C B- \\
153 \\
\end{array}$ & $\begin{array}{c}P C B- \\
138 \\
\end{array}$ & $\begin{array}{r}P C B \\
180 \\
\end{array}$ \\
\hline \multirow{12}{*}{ 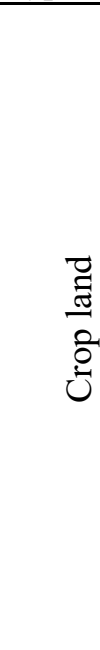 } & \multirow{4}{*}{ Winter } & \multirow{2}{*}{1} & I & 3.1 & 1.4 & 0.7 & 0.1 & 0.2 & 0.1 \\
\hline & & & II & 2.7 & 1.1 & 0.6 & 0.2 & 0.6 & 0.0 \\
\hline & & \multirow{2}{*}{2} & I & 1.8 & 0.5 & 0.4 & 0.1 & 0.1 & 0.2 \\
\hline & & & II & 1.2 & 0.3 & 0.2 & 0.0 & 0.1 & 0.0 \\
\hline & \multirow{4}{*}{$\begin{array}{c}\text { Pre- } \\
\text { monsoon }\end{array}$} & \multirow{2}{*}{3} & I & 2.4 & 1.0 & 0.6 & 0.2 & 0.2 & 0.1 \\
\hline & & & II & 2.4 & 0.4 & 0.3 & 0.0 & 0.0 & 0.0 \\
\hline & & \multirow{2}{*}{4} & I & 3.1 & 1.3 & 0.8 & 1.7 & 0.3 & 0.1 \\
\hline & & & II & 1.5 & 0.7 & 0.5 & 0.7 & 0.2 & 0.0 \\
\hline & \multirow{4}{*}{ Monsoon } & \multirow{2}{*}{5} & I & 1.9 & 1.0 & 0.4 & 1.4 & 0.1 & 0.0 \\
\hline & & & II & 2.1 & 1.2 & 0.6 & 1.3 & 0.2 & 0.0 \\
\hline & & 6 & I & 2.5 & 1.0 & 0.6 & 0.2 & 0.3 & 0.0 \\
\hline & & 0 & II & 3.3 & 0.7 & 0.4 & 0.2 & 0.0 & 0.0 \\
\hline \multirow{11}{*}{ 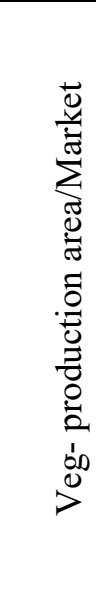 } & \multirow{4}{*}{ Winter } & \multirow{2}{*}{1} & I & 3.5 & 1.8 & 0.9 & 0.4 & 0.8 & 0.0 \\
\hline & & & II & 4.1 & 1.5 & 0.9 & 0.3 & 0.7 & 0.0 \\
\hline & & \multirow{2}{*}{2} & I & 6.4 & 2.1 & 1.1 & 0.5 & 0.5 & 0.1 \\
\hline & & & II & 6.5 & 1.7 & 1.0 & 0.4 & 0.6 & 0.1 \\
\hline & \multirow{3}{*}{$\begin{array}{c}\text { Pre- } \\
\text { monsoon }\end{array}$} & \multirow{2}{*}{3} & I & 4.8 & 3.4 & 1.6 & 0.7 & 0.9 & 0.2 \\
\hline & & & II & 5.7 & 3.5 & 1.5 & 0.6 & 1.1 & 0.3 \\
\hline & & 4 & I & 4.2 & 2.0 & 1.2 & 1.8 & 0.7 & 0.2 \\
\hline & \multirow{4}{*}{ Monsoon } & \multirow{2}{*}{5} & I & 8.4 & 6.4 & 3.6 & 3.1 & 1.5 & 0.3 \\
\hline & & & II & 6.4 & 5.6 & 3.3 & 2.0 & 1.2 & 0.3 \\
\hline & & \multirow{2}{*}{6} & I & 5.9 & 2.9 & 1.8 & 0.7 & 0.7 & 0.2 \\
\hline & & & II & 7.1 & 4.2 & 2.2 & 1.2 & 0.3 & 0.3 \\
\hline \multirow{12}{*}{ 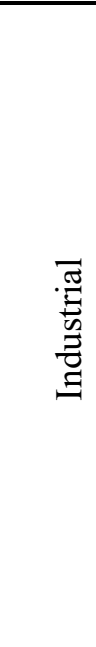 } & \multirow{4}{*}{ Winter } & \multirow{2}{*}{1} & I & 2.1 & 0.9 & 0.3 & 1.8 & 3.3 & 0.1 \\
\hline & & & II & 3.2 & 0.7 & 0.4 & 0.1 & 0.2 & 0.0 \\
\hline & & \multirow{2}{*}{2} & I & 3.8 & 2.2 & 0.7 & 0.4 & 0.4 & 0.1 \\
\hline & & & II & 4.5 & 2.2 & 0.8 & 0.3 & 0.3 & 0.1 \\
\hline & \multirow{4}{*}{$\begin{array}{c}\text { Pre- } \\
\text { monsoon }\end{array}$} & \multirow{2}{*}{3} & I & 5.3 & 4.4 & 4.0 & 1.7 & 2.1 & 0.3 \\
\hline & & & II & 5.6 & 3.8 & 3.5 & 1.5 & 1.7 & 0.2 \\
\hline & & \multirow{2}{*}{4} & I & 5.3 & 2.6 & 1.2 & 2.1 & 0.6 & 0.2 \\
\hline & & & II & 4.6 & 1.5 & 0.9 & 2.0 & 0.4 & 0.1 \\
\hline & \multirow{4}{*}{ Monsoon } & 5 & I & 4.4 & 2.8 & 2.0 & 1.5 & 0.9 & 0.2 \\
\hline & & J & II & 9.6 & 4.5 & 3.5 & 2.2 & 1.6 & 0.2 \\
\hline & & 6 & I & 19.4 & 3.8 & 1.2 & 0.7 & 0.5 & 0.1 \\
\hline & & 0 & II & 19.6 & 21.7 & 2.4 & 1.9 & 1.5 & 0.3 \\
\hline
\end{tabular}




\begin{tabular}{|c|c|c|c|c|c|c|c|c|c|}
\hline \multirow{10}{*}{ 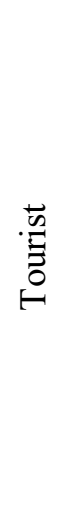 } & \multirow{3}{*}{ Winter } & \multirow{2}{*}{1} & I & 9.7 & 1.7 & 0.8 & 0.7 & 0.4 & 0.1 \\
\hline & & & II & 5.4 & 0.9 & 0.4 & 1.5 & 0.2 & 0.0 \\
\hline & & 2 & I & 3.8 & 0.6 & 0.5 & 0.4 & 0.1 & 0.0 \\
\hline & \multirow{3}{*}{$\begin{array}{c}\text { Pre- } \\
\text { monsoon }\end{array}$} & \multirow{2}{*}{3} & I & 4.5 & 0.7 & 0.4 & 0.6 & 0.0 & 0.0 \\
\hline & & & II & 4.3 & 2.0 & 0.9 & 0.7 & 0.4 & 0.1 \\
\hline & & 4 & I & 5.1 & 1.9 & 1.2 & 1.9 & 0.5 & 0.2 \\
\hline & \multirow{4}{*}{ Monsoon } & \multirow{2}{*}{5} & I & 6.7 & 1.9 & 1.2 & 1.8 & 0.5 & 0.0 \\
\hline & & & II & 4.6 & 1.9 & 1.2 & 1.7 & 0.4 & 0.1 \\
\hline & & \multirow{2}{*}{6} & I & 6.0 & 2.6 & 1.3 & 0.7 & 0.6 & 0.2 \\
\hline & & & II & 5.2 & 2.0 & 1.1 & 0.3 & 0.4 & 0.0 \\
\hline \multirow{12}{*}{ 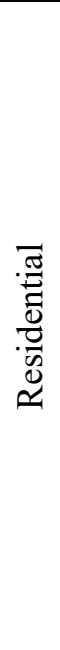 } & \multirow{4}{*}{ Winter } & \multirow{2}{*}{1} & I & 1.8 & 0.7 & 0.5 & 0.2 & 0.4 & 0.0 \\
\hline & & & II & 7.4 & 3.0 & 1.7 & 0.7 & 0.8 & 0.1 \\
\hline & & \multirow{2}{*}{2} & I & 6.0 & 2.9 & 1.4 & 0.6 & 0.8 & 0.2 \\
\hline & & & II & 5.0 & 1.4 & 0.7 & 0.3 & 0.5 & 0.1 \\
\hline & \multirow{4}{*}{$\begin{array}{c}\text { Pre- } \\
\text { monsoon }\end{array}$} & \multirow{2}{*}{3} & I & 13.3 & 6.4 & 2.5 & 1.8 & 1.4 & 0.5 \\
\hline & & & II & 12.8 & 6.6 & 2.5 & 1.7 & 1.3 & 0.5 \\
\hline & & \multirow{2}{*}{4} & I & 12.4 & 3.9 & 1.5 & 1.9 & 0.9 & 0.2 \\
\hline & & & II & 11.2 & 4.7 & 1.7 & 2.0 & 0.8 & 0.2 \\
\hline & \multirow{4}{*}{ Monsoon } & \multirow{2}{*}{5} & I & 14.2 & 7.3 & 2.6 & 2.4 & 1.1 & 0.3 \\
\hline & & & II & 10.4 & 5.1 & 2.0 & 2.1 & 1.1 & 0.2 \\
\hline & & \multirow{2}{*}{6} & I & 12.3 & 6.5 & 4.8 & 3.1 & 2.4 & 0.3 \\
\hline & & & II & 13.7 & 7.4 & 5.7 & 3.7 & 3.2 & 0.4 \\
\hline \multirow{12}{*}{ 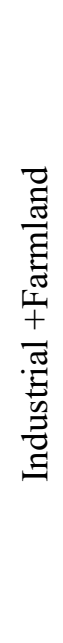 } & \multirow{4}{*}{ Winter } & \multirow{2}{*}{1} & I & 9.4 & 2.5 & 1.0 & 0.7 & 0.6 & 0.3 \\
\hline & & & II & 12.6 & 2.5 & 0.9 & 0.6 & 0.4 & 0.2 \\
\hline & & \multirow{2}{*}{2} & I & 7.5 & 2.5 & 0.8 & 0.5 & 0.5 & 0.2 \\
\hline & & & II & 7.5 & 2.0 & 0.7 & 0.6 & 0.6 & 0.2 \\
\hline & \multirow{4}{*}{$\begin{array}{c}\text { Pre- } \\
\text { monsoon }\end{array}$} & \multirow{2}{*}{3} & I & 10.6 & 3.9 & 1.3 & 1.2 & 0.7 & 0.4 \\
\hline & & & II & 10.9 & 3.7 & 1.3 & 1.3 & 0.7 & 0.3 \\
\hline & & \multirow{2}{*}{4} & I & 22.7 & 5.6 & 1.3 & 1.6 & 0.6 & 0.3 \\
\hline & & & II & 8.6 & 2.9 & 1.6 & 1.8 & 1.1 & 0.7 \\
\hline & \multirow{4}{*}{ Monsoon } & \multirow{2}{*}{5} & I & 15.1 & 3.8 & 1.5 & 3.4 & 0.8 & 0.4 \\
\hline & & & II & 13.1 & 4.3 & 1.6 & 1.6 & 1.0 & 0.4 \\
\hline & & 6 & I & 13.2 & 4.4 & 1.6 & 1.1 & 0.9 & 0.2 \\
\hline & & 0 & II & 19.9 & 6.2 & 2.0 & 1.6 & 0.9 & 0.3 \\
\hline
\end{tabular}




\begin{tabular}{|c|c|c|c|c|c|c|c|c|c|}
\hline $\begin{array}{l}\text { Site } \\
\text { type }\end{array}$ & Seasons & & ling & $\begin{array}{c}P C B- \\
28\end{array}$ & $\begin{array}{c}P C B- \\
52\end{array}$ & $\begin{array}{c}P C B- \\
101\end{array}$ & $\begin{array}{c}P C B- \\
153\end{array}$ & $\begin{array}{c}P C B- \\
138\end{array}$ & $\begin{array}{c}P C B- \\
180\end{array}$ \\
\hline \multirow{11}{*}{$\begin{array}{l}\bar{\Xi} \\
\frac{\vec{t}}{2} \\
\overline{0} \\
\tilde{U}\end{array}$} & \multirow{3}{*}{ Winter } & \multirow{2}{*}{1} & I & 1.3 & 0.4 & 0.3 & 0.5 & 0.0 & 0.0 \\
\hline & & & II & 1.0 & 0.2 & 0.2 & 1.3 & 0.0 & 0.0 \\
\hline & & 2 & I & 1.3 & 0.4 & 0.2 & 0.4 & 0.0 & 0.0 \\
\hline & \multirow{4}{*}{$\begin{array}{c}\text { Pre- } \\
\text { monsoon }\end{array}$} & \multirow{2}{*}{3} & I & 2.0 & 1.1 & 0.5 & 0.5 & 0.1 & 0.0 \\
\hline & & & II & 1.4 & 0.5 & 0.2 & 0.5 & 0.0 & 0.0 \\
\hline & & \multirow{2}{*}{4} & I & 1.1 & 0.5 & 0.4 & 0.5 & 0.0 & 0.0 \\
\hline & & & II & 2.0 & 0.5 & 0.3 & 0.6 & 0.1 & 0.0 \\
\hline & \multirow{4}{*}{ Monsoon } & \multirow{2}{*}{5} & I & 5.4 & 1.0 & 0.5 & 0.7 & 0.0 & 0.0 \\
\hline & & & II & 2.0 & 0.8 & 0.5 & 0.7 & 0.0 & 0.0 \\
\hline & & 6 & I & 1.4 & 0.7 & 0.4 & 0.1 & 0.2 & 0.1 \\
\hline & & 0 & II & 0.8 & 0.3 & 0.2 & 0.0 & 0.0 & 0.0 \\
\hline \multirow{11}{*}{ 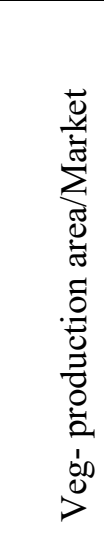 } & \multirow{4}{*}{ Winter } & \multirow{2}{*}{1} & I & 1.9 & 0.9 & 0.7 & 0.9 & 0.5 & 0.1 \\
\hline & & & II & 1.5 & 0.5 & 0.4 & 0.6 & 0.4 & 0.0 \\
\hline & & \multirow{2}{*}{2} & I & 1.0 & 0.5 & 0.6 & 0.5 & 0.0 & 0.2 \\
\hline & & & II & 1.6 & 0.6 & 0.6 & 0.6 & 0.4 & 0.2 \\
\hline & \multirow{3}{*}{$\begin{array}{c}\text { Pre- } \\
\text { monsoon }\end{array}$} & \multirow{2}{*}{3} & I & 1.6 & 0.7 & 0.7 & 0.9 & 0.4 & 0.2 \\
\hline & & & II & 3.3 & 1.3 & 0.7 & 1.6 & 0.3 & 0.1 \\
\hline & & 4 & I & 1.9 & 0.9 & 1.0 & 1.1 & 0.7 & 0.2 \\
\hline & \multirow{4}{*}{ Monsoon } & \multirow{2}{*}{5} & I & 3.1 & 2.1 & 0.3 & 0.4 & 0.3 & 0.1 \\
\hline & & & II & 2.4 & 1.5 & 0.1 & 0.3 & 0.2 & 0.1 \\
\hline & & \multirow{2}{*}{6} & I & 1.8 & 0.8 & 0.6 & 0.6 & 0.3 & 0.1 \\
\hline & & & II & 1.5 & 0.6 & 0.5 & 0.3 & 0.2 & 0.1 \\
\hline \multirow{12}{*}{ 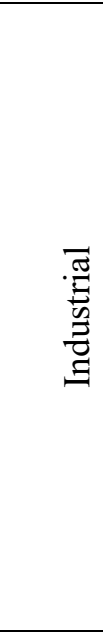 } & \multirow{4}{*}{ Winter } & \multirow{2}{*}{1} & I & 2.5 & 1.0 & 0.6 & 0.2 & 0.2 & 0.0 \\
\hline & & & II & 1.2 & 0.5 & 0.3 & 0.0 & 0.0 & 0.0 \\
\hline & & \multirow{2}{*}{2} & I & 5.7 & 1.8 & 0.8 & 0.4 & 0.3 & 0.0 \\
\hline & & & II & 17.3 & 5.8 & 2.9 & 1.0 & 1.0 & 0.2 \\
\hline & \multirow{4}{*}{$\begin{array}{c}\text { Pre- } \\
\text { monsoon }\end{array}$} & \multirow{2}{*}{3} & I & 5.3 & 1.3 & 0.1 & 0.2 & 0.2 & 0.1 \\
\hline & & & II & 6.2 & 1.6 & 0.1 & 0.3 & 0.3 & 0.1 \\
\hline & & \multirow{2}{*}{4} & I & 1.2 & 0.5 & 0.3 & 0.4 & 0.2 & 0.0 \\
\hline & & & II & 2.0 & 0.4 & 0.4 & 0.5 & 0.1 & 0.0 \\
\hline & & 5 & I & 0.7 & 0.3 & 0.2 & 0.8 & 0.1 & 0.0 \\
\hline & Monsoon & $\mathrm{J}$ & II & 0.7 & 0.3 & 0.2 & 1.1 & 0.1 & 0.0 \\
\hline & Monsoon & 6 & I & 1.5 & 0.9 & 0.4 & 0.1 & 0.1 & 0.1 \\
\hline & & 0 & II & 1.9 & 1.1 & 0.5 & 0.2 & 0.2 & 0.0 \\
\hline & & 1 & I & 0.9 & 0.3 & 0.1 & 0.8 & 0.3 & 0.0 \\
\hline & Winter & 2 & I & 1.8 & 0.4 & 0.2 & 0.1 & 0.2 & 0.0 \\
\hline$\stackrel{\vec{n}}{\Xi}$ & & 2 & II & 1.5 & 0.4 & 0.2 & 0.0 & 0.0 & 0.0 \\
\hline$\stackrel{\Xi}{\ominus}$ & & 3 & I & 2.2 & 0.5 & 0.4 & 5.6 & 0.2 & 0.1 \\
\hline & $\begin{array}{c}\text { Pre- } \\
\text { monsoon }\end{array}$ & $J$ & II & 1.4 & 0.5 & 0.3 & 1.6 & 0.2 & 0.0 \\
\hline & & 4 & I & 1.3 & 0.6 & 0.6 & 0.6 & 0.3 & 0.1 \\
\hline
\end{tabular}




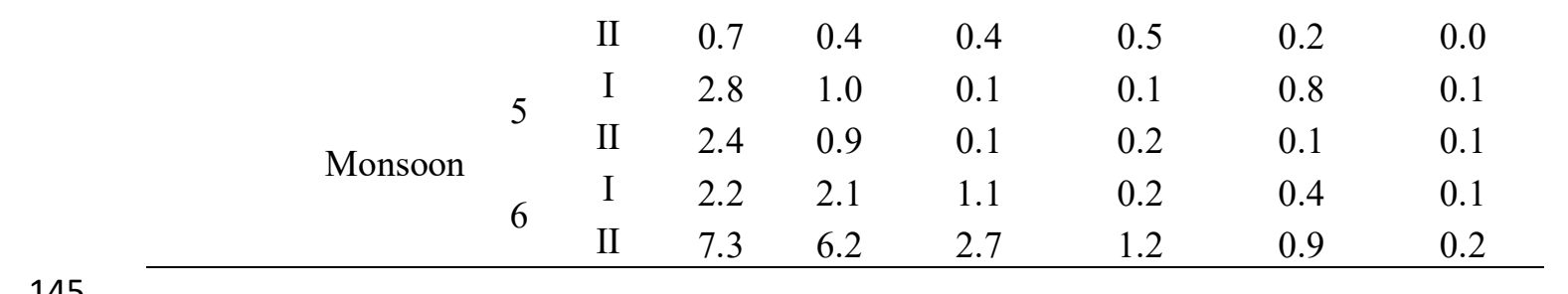

Table SI-6c. Site specific concentrations $\left(\mathrm{pg} / \mathrm{m}^{3}\right)$ of PCBs in different urban sites of Hetauda

\begin{tabular}{|c|c|c|c|c|c|c|c|c|c|}
\hline $\begin{array}{l}\text { Site } \\
\text { type }\end{array}$ & Seasons & & & $\begin{array}{c}P C B- \\
28\end{array}$ & $\begin{array}{c}P C B- \\
52\end{array}$ & $\begin{array}{c}P C B- \\
101\end{array}$ & $\begin{array}{c}P C B- \\
153\end{array}$ & $\begin{array}{c}P C B- \\
138\end{array}$ & $\begin{array}{c}P C B- \\
180\end{array}$ \\
\hline \multirow{10}{*}{$\begin{array}{l}\overrightarrow{\widetilde{a}} \\
\frac{\vec{\sigma}}{2} \\
\frac{0}{0}\end{array}$} & \multirow{4}{*}{ Winter } & \multirow{2}{*}{1} & I & 3.2 & 1.2 & 0.0 & 0.2 & 0.2 & 0.2 \\
\hline & & & II & 3.6 & 0.6 & 0.0 & 0.1 & 0.2 & 0.0 \\
\hline & & \multirow{2}{*}{2} & I & 1.5 & 0.9 & 0.6 & 0.2 & 0.2 & 0.1 \\
\hline & & & II & 2.0 & 0.9 & 0.3 & 0.2 & 0.2 & 0.0 \\
\hline & \multirow{4}{*}{$\begin{array}{c}\text { Pre- } \\
\text { monsoon }\end{array}$} & \multirow{2}{*}{3} & I & 1.8 & 0.7 & 0.4 & 0.2 & 0.2 & 0.0 \\
\hline & & & II & 2.5 & 0.9 & 0.9 & 0.3 & 0.4 & 0.1 \\
\hline & & \multirow{2}{*}{4} & I & 0.9 & 0.4 & 0.5 & 0.4 & 0.2 & 0.0 \\
\hline & & & II & 1.0 & 0.2 & 0.1 & 0.1 & 0.1 & 0.0 \\
\hline & \multirow{2}{*}{ Monsoon } & \multirow{2}{*}{5} & I & 0.8 & 0.5 & 0.7 & 0.2 & 0.2 & 0.0 \\
\hline & & & II & 4.7 & 3.6 & 1.2 & 1.7 & 1.9 & 0.7 \\
\hline \multirow{10}{*}{ 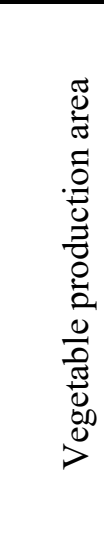 } & \multirow{4}{*}{ Winter } & \multirow{2}{*}{1} & I & 5.9 & 3.4 & 0.9 & 0.6 & 0.8 & 0.3 \\
\hline & & & II & 10.6 & 1.4 & 0.0 & 0.1 & 0.0 & 0.2 \\
\hline & & \multirow{2}{*}{2} & I & 10.9 & 3.0 & 0.9 & 0.3 & 0.4 & 0.2 \\
\hline & & & II & 4.0 & 1.5 & 0.0 & 0.1 & 0.7 & 0.6 \\
\hline & \multirow{4}{*}{$\begin{array}{c}\text { Pre- } \\
\text { monsoon }\end{array}$} & \multirow{2}{*}{3} & I & 6.0 & 4.3 & 0.9 & 0.9 & 2.2 & 0.5 \\
\hline & & & II & 3.1 & 2.5 & 1.4 & 0.5 & 1.4 & 0.4 \\
\hline & & \multirow{2}{*}{4} & I & 1.6 & 1.5 & 1.8 & 0.3 & 0.9 & 0.3 \\
\hline & & & II & 0.7 & 1.0 & 0.6 & 0.1 & 1.5 & 0.8 \\
\hline & \multirow{2}{*}{ Monsoon } & \multirow{2}{*}{5} & I & 2.9 & 1.7 & 0.8 & 0.3 & 0.5 & 0.3 \\
\hline & & & II & 2.4 & 1.5 & 0.7 & 0.4 & 0.3 & 0.1 \\
\hline \multirow{10}{*}{ 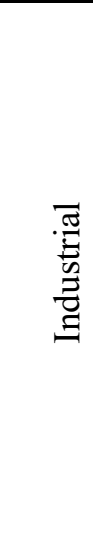 } & \multirow{4}{*}{ Winter } & \multirow{2}{*}{1} & I & 4.2 & 2.0 & 1.1 & 1.2 & 1.9 & 0.4 \\
\hline & & & II & 3.8 & 0.9 & 1.2 & 1.1 & 0.8 & 0.3 \\
\hline & & \multirow{2}{*}{2} & I & 1.4 & 0.5 & 0.3 & 0.2 & 0.4 & 0.1 \\
\hline & & & II & 3.2 & 1.5 & 0.3 & 0.7 & 0.7 & 0.3 \\
\hline & \multirow{4}{*}{$\begin{array}{c}\text { Pre- } \\
\text { monsoon }\end{array}$} & \multirow{2}{*}{3} & I & 5.2 & 1.3 & 1.4 & 0.6 & 0.8 & 0.2 \\
\hline & & & II & 1.4 & 0.8 & 1.0 & 0.5 & 0.6 & 0.1 \\
\hline & & \multirow{2}{*}{4} & I & 5.6 & 1.0 & 0.4 & 0.2 & 0.4 & 0.1 \\
\hline & & & II & 6.8 & 1.8 & 0.8 & 0.5 & 0.7 & 0.3 \\
\hline & \multirow{2}{*}{ Monsoon } & \multirow{2}{*}{5} & I & 2.8 & 2.5 & 1.1 & 1.1 & 1.0 & 0.3 \\
\hline & & & II & 2.1 & 1.7 & 0.7 & 0.7 & 0.6 & 0.2 \\
\hline
\end{tabular}


Table SI-7. Comparison of current levels $\left(\mathrm{pg} / \mathrm{m}^{3}\right)$ of various POPs with different tropical/subtropical urban sites

\begin{tabular}{|c|c|c|c|c|c|c|c|c|c|}
\hline Places & $\mathrm{o}, \mathrm{p}^{\prime}-\mathrm{DDT}$ & $\mathrm{p}, \mathrm{p}^{\prime}$-DDT & $\mathrm{p}, \mathrm{p}^{\prime}-\mathrm{DDE}$ & $\alpha-\mathrm{HCH}$ & $\gamma-\mathrm{HCH}$ & $\alpha$-endo & $\beta$-endo & $\sum \mathrm{PCBs}$ & Sampling time \\
\hline \multicolumn{10}{|c|}{ This study } \\
\hline Kathmandu* & $3-90$ & $3-145$ & $4-187$ & $3-73$ & $2-229$ & BDL-16 & BDL- 35 & $2-47$ & Aug, 2014 - Aug, 2015 \\
\hline Pokhara* & $3-69$ & $6-621$ & $9-364$ & $3-14$ & $5-39$ & BDL-13 & $1-12$ & $1-28$ & Aug, 2014 - Aug, 2015 \\
\hline Hetauda* & $1-7$ & $2-49$ & $2-22$ & $1-11$ & $3-3326$ & $1-5$ & $3-36$ & $2-16$ & Oct, 2015 - Nov, 2016 \\
\hline \multicolumn{10}{|c|}{ GAPs study $^{\mathbf{a}}$} \\
\hline Chengdu, Chi & & $\mathrm{BDL}$ & $\mathrm{BDL}-56$ & $145-176$ & $68-142$ & $8-47$ & BDL-59 & $187-249$ & Jan-Jun2005 \\
\hline Kuwait city, $\mathrm{K}$ & wait** & 131 & $22-58$ & $1-13$ & $1-17$ & $76-168$ & BDL-16 & $86-497$ & Jan-Sept 2005 \\
\hline Manila, Philip & ines** & 190 & $14-45$ & BDL-1 & BDL-15 & $13-66$ & BDL-4 & $629-2826$ & Jan-Sept 2005 \\
\hline Izmir, Turkey & & BDL & $60-46$ & $18-30$ & $13-18$ & $494-1352$ & $46-464$ & $174-287$ & Jan-Jun2005 \\
\hline Seoul, Korea* & & & 34 & 84 & 43 & 4411 & 957 & 397 & Jun-Sept 2005 \\
\hline Malawi, Afric & & & BDL & BDL & 9 & 162 & 10 & $\mathrm{BDL}$ & Mar-June 2005 \\
\hline \multicolumn{10}{|c|}{ Mexico $^{b}$} \\
\hline Mexico city & 17 & ND & 21 & 8.9 & 49 & 320 & 68 & & $2005-2006$ \\
\hline Chihuahua & 1.7 & ND & 25 & 5.9 & 11 & 351 & 95 & & $2005-2006$ \\
\hline $\begin{array}{l}\text { San Luis } \\
\text { Potosi }\end{array}$ & 1.4 & ND & 13 & 9.4 & 16 & 260 & 40 & & $2005-2006$ \\
\hline \multicolumn{10}{|c|}{ Nepal $^{\mathrm{c}}$} \\
\hline Kathmandu & $9-15$ & $8-62$ & $4-83$ & $3-51$ & $4-272$ & $6-30$ & $6-15$ & & Aug-Oct, 2014 \\
\hline Pokhara & $10-18$ & $8-120$ & $10-25$ & $7-13$ & $4-36$ & $8-34$ & $6-14$ & & Aug-Oct, 2014 \\
\hline Birgunj & $16-86$ & $105-1170$ & $41-180$ & $8-36$ & $24-243$ & $8-46$ & $7-25$ & & Aug-Oct, 2014 \\
\hline Biratnagar & $25-136$ & $236-3340$ & $29-1760$ & $9-21$ & $34-138$ & $12-20$ & $14-31$ & & Aug-Oct, 2014 \\
\hline
\end{tabular}

All the studies used PUF-PAS

$150 \quad{ }^{a}$ Pozo et al., 2009; ${ }^{b}$ Wong et al., 2009; ${ }^{c}$ Yadav et al., 2017

$151 * \sum 6 P C B S$

$152 * * \sum 48 P C B S$ 
155

\begin{tabular}{|c|c|c|c|c|c|}
\hline & Minimum & Maximum & Mean & SD & $\mathrm{H} / \mathrm{L}$ \\
\hline o,p'-DDT & 0.9 & 89.5 & 20.4 & 19.0 & 95.8 \\
\hline $\mathrm{p}, \mathrm{p}$-DDT & 2.4 & 620.7 & 42.7 & 71.8 & 263.8 \\
\hline$o, p^{\prime}-\mathrm{DDE}$ & 0.1 & 12.3 & 2.8 & 2.4 & 126.9 \\
\hline $\mathrm{p}, \mathrm{p}^{\prime}-\mathrm{DDE}$ & 2.2 & 364.1 & 37.6 & 50.7 & 162.4 \\
\hline $\mathrm{o}, \mathrm{p}^{\prime}-\mathrm{DDD}$ & 0.0 & 4.9 & 1.0 & 1.1 & \\
\hline p,p'-DDD & 0.0 & 19.7 & 3.3 & 3.0 & \\
\hline$\sum$ DDTs & 8.7 & 1020.3 & 107.9 & 133.0 & 117.1 \\
\hline$\alpha-\mathrm{HCH}$ & 1.2 & 72.9 & 11.2 & 12.4 & 58.3 \\
\hline$\beta-\mathrm{HCH}$ & 0.0 & 16.0 & 2.7 & 3.1 & \\
\hline$\gamma-\mathrm{HCH}$ & 2.5 & 3326.3 & 155.7 & 560.7 & 1351.3 \\
\hline$\delta-\mathrm{HCH}$ & 0.0 & 9.6 & 2.0 & 2.0 & \\
\hline$\sum \mathrm{HCHs}$ & 5.3 & 3334.6 & 171.6 & 559.7 & 632.4 \\
\hline HCB & 5.8 & 347.0 & 33.1 & 41.0 & 59.7 \\
\hline$\alpha$-endo & 0.0 & 15.7 & 2.0 & 2.6 & \\
\hline$\beta$-endo & 0.0 & 35.6 & 7.9 & 6.8 & \\
\hline ¿endo & 0.0 & 51.1 & 9.9 & 8.2 & \\
\hline PCB-28 & 0.7 & 22.7 & 5.0 & 4.5 & 33.6 \\
\hline PCB-52 & 0.2 & 21.7 & 2.1 & 2.4 & 119.6 \\
\hline PCB-101 & 0.0 & 5.7 & 1.0 & 0.9 & \\
\hline PCB-153 & 0.0 & 5.6 & 0.9 & 0.8 & \\
\hline PCB-138 & 0.0 & 3.3 & 0.6 & 0.6 & \\
\hline PCB-180 & 0.0 & 0.8 & 0.1 & 0.2 & \\
\hline$\sum \mathrm{PCBs}$ & 1.4 & 47.4 & 9.7 & 8.1 & 35.1 \\
\hline
\end{tabular}




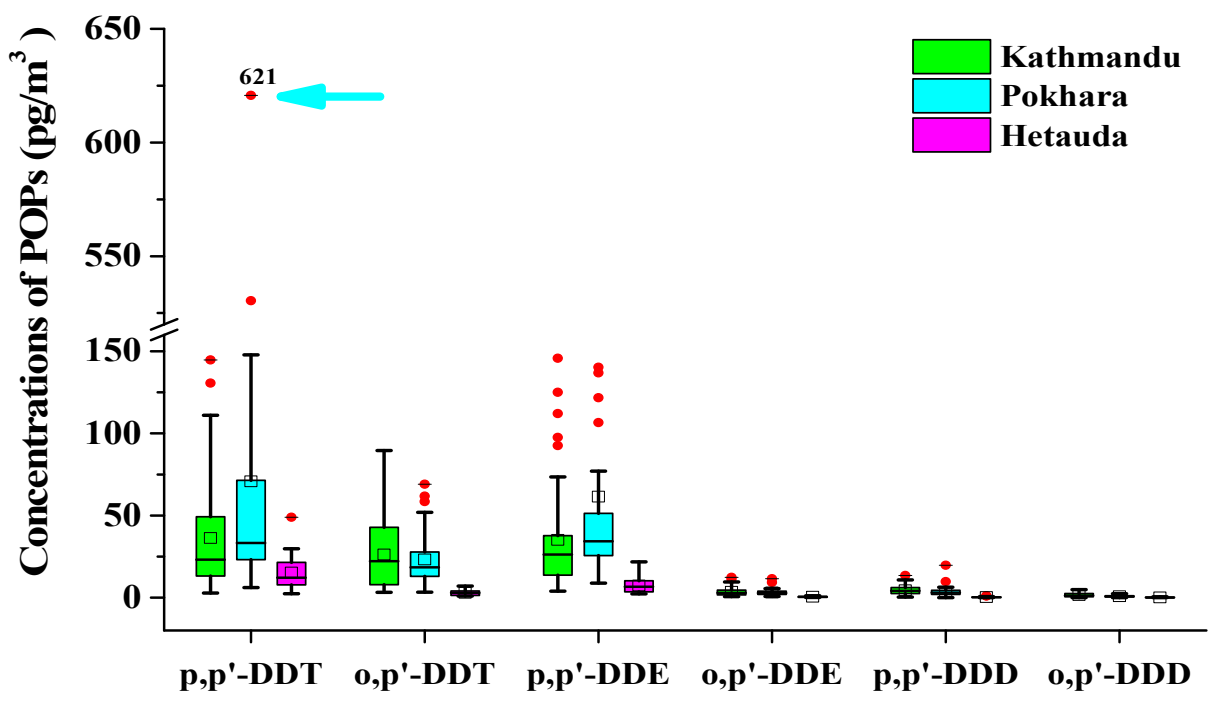

159 Figure SI-3. Box and whisker plot to show distribution of different isomers of DDT and 160 its metabolites in Kathmandu Pokhara and Hetauda(Lower and upper limits of whisker 161 indicate minimum and maximum, Lower and upper limits of the box indicate $25^{\text {th }}$ and $75^{\text {th }}$ 162 percentiles, horizontal line in the box indicates median, small square in the box represents mean, 163 red circle denotes outlier)

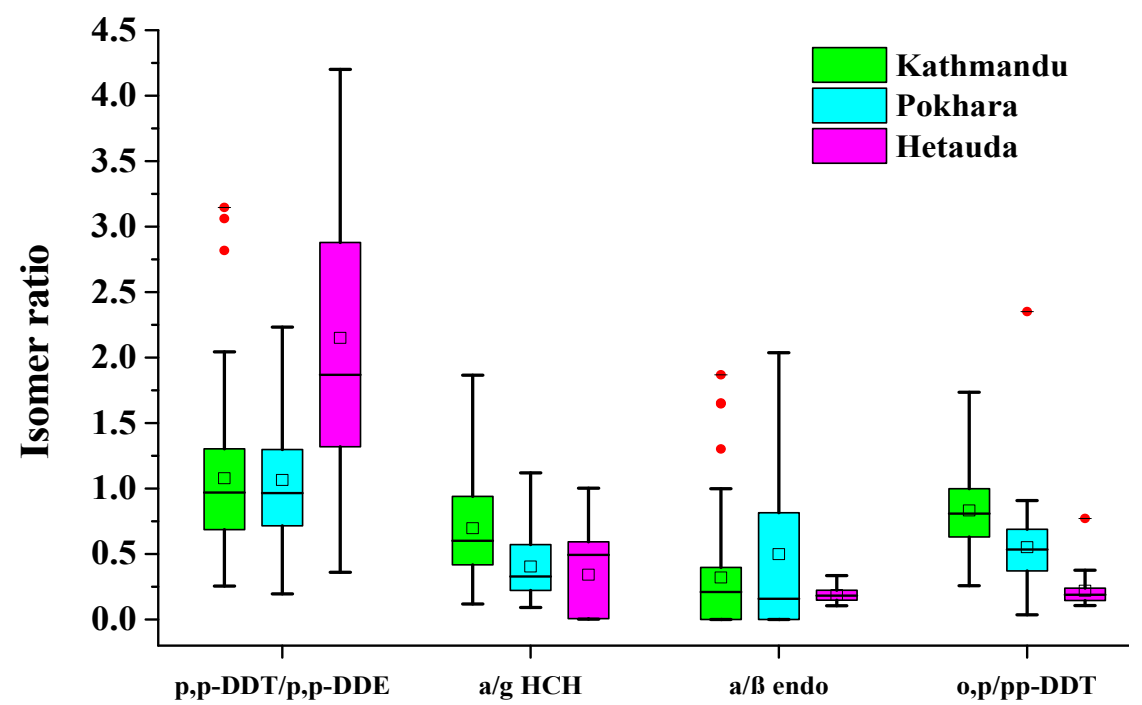

Figure SI-4. Isomers/ metabolites ratios of selected OCPs to predict source type(Lower and upper limits of whisker indicate minimum and maximum, Lower and upper limits of the box indicate $25^{\text {th }}$ and $75^{\text {th }}$ percentiles, horizontal line in the box indicates median, small square in the box represents mean, red circle denotes outlier) 


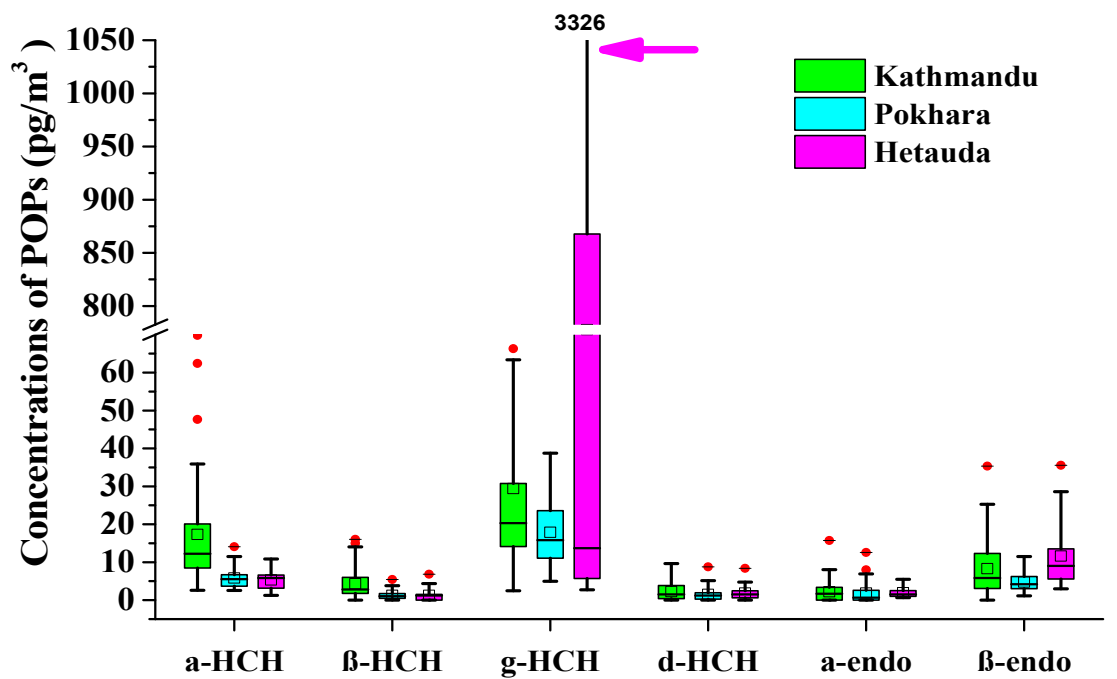

170 Figure SI-5. Box and whisker plot to show distribution of different isomers of HCH and endosulfan 171 in Kathmandu Pokhara and Hetauda(Lower and upper limits of whisker indicate minimum and 172 maximum, Lower and upper limits of the box indicate $25^{\text {th }}$ and $75^{\text {th }}$ percentiles, horizontal line inside the 173 box indicates median, small square in the box represents mean, red circle denotes outlier)

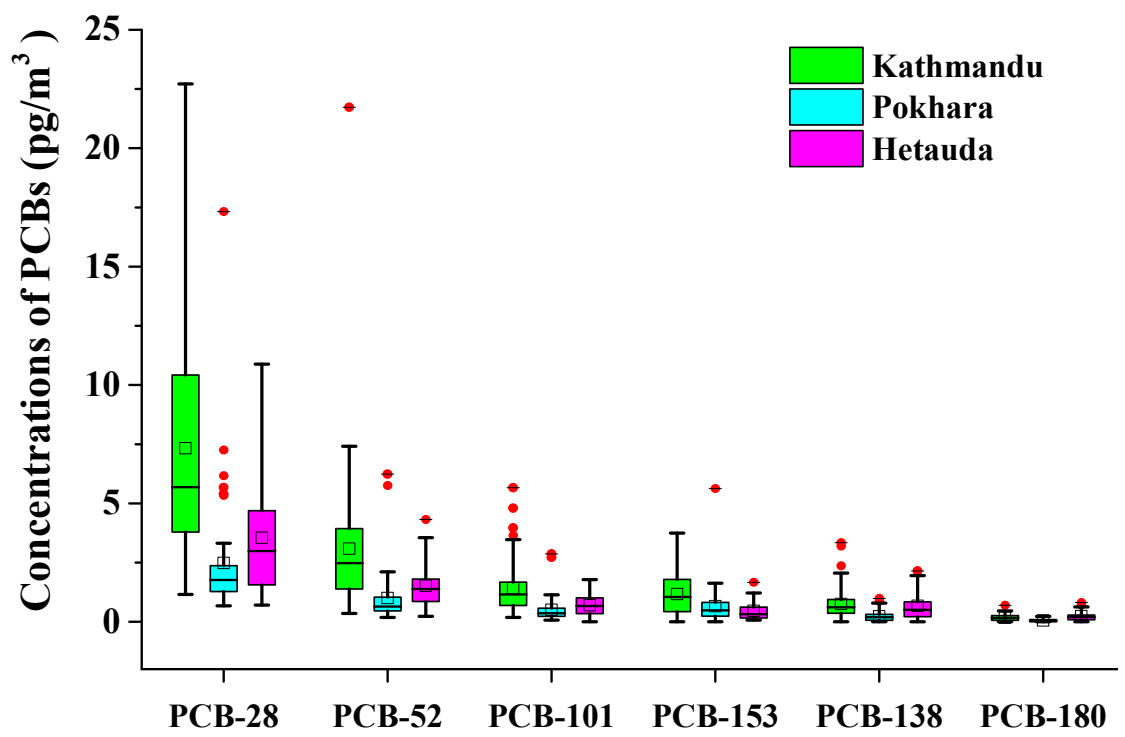

176 Figure SI-6. Box and whisker plot to show distribution of different congeners of PCBs in 177 Kathmandu Pokhara and Hetauda(Lower and upper limits of whisker indicate minimum and 178 maximum, Lower and upper limits of the box indicate $25^{\text {th }}$ and $75^{\text {th }}$ percentiles, horizontal line in the box 179 indicates median, small square in the box represents mean, red circle denotes outlier) 


\begin{tabular}{lcccccccccc}
\hline & $o, p^{\prime}-D D T$ & $p, p^{\prime}-D D T$ & $o, p^{\prime}-D D E$ & $p, p^{\prime}-D D E$ & $\alpha-H C H$ & $\gamma-H C H$ & HCB & $\alpha$-endo & $\beta$-endo & PCBs \\
\hline Kathmandu & 0.40 & $\mathbf{0 . 0 1}$ & $\mathbf{0 . 0 1}$ & $\mathbf{0 . 0 0}$ & 0.15 & $\mathbf{0 . 0 5}$ & $\mathbf{0 . 0 0}$ & 0.37 & 0.21 & $\mathbf{0 . 0 0}$ \\
Pokhara & 0.65 & 0.27 & 0.34 & 0.18 & 0.29 & 0.08 & 0.49 & 0.17 & 0.13 & 0.54 \\
Hetauda & 0.14 & 0.17 & $\mathbf{0 . 0 2}$ & $\mathbf{0 . 0 2}$ & 0.69 & $\mathbf{0 . 0 0}$ & 0.82 & 0.18 & 0.13 & $\mathbf{0 . 0 3}$ \\
\hline
\end{tabular}

183 
192
Table SI-10. Significant differences $(\mathbf{P}<0.05$, Tukey's Test) in OCPs concentrations among the sites in Kathmandu (only the chemicals with variations have been shown)

\begin{tabular}{|c|c|c|c|c|c|c|}
\hline & & $\mathrm{p}, \mathrm{p}^{\prime}-\mathrm{DDT}$ & $\mathrm{p}, \mathrm{p}^{\prime}-\mathrm{DDE}$ & $\gamma-\mathrm{HCH}$ & HCB & PCB \\
\hline \multirow[t]{5}{*}{ K1 } & K2 & 0.03 & 0.00 & 0.05 & 1.00 & 0.32 \\
\hline & K3 & 1.00 & 0.90 & 0.98 & 0.00 & 0.06 \\
\hline & K4 & 1.00 & 0.80 & 0.64 & 0.99 & 0.80 \\
\hline & K5 & 1.00 & 0.92 & 0.99 & 1.00 & 0.00 \\
\hline & K6 & 1.00 & 0.96 & 0.99 & 0.99 & 0.01 \\
\hline \multirow[t]{4}{*}{ K2 } & K3 & 0.04 & 0.01 & 0.17 & 0.00 & 0.95 \\
\hline & K4 & 0.01 & 0.02 & 0.66 & 0.99 & 0.96 \\
\hline & K5 & 0.05 & 0.01 & 0.16 & 1.00 & 0.37 \\
\hline & K6 & 0.04 & 0.01 & 0.15 & 0.99 & 0.39 \\
\hline \multirow[t]{3}{*}{ K3 } & K4 & 0.99 & 1.00 & 0.93 & 0.00 & 0.52 \\
\hline & K5 & 1.00 & 1.00 & 1.00 & 0.00 & 0.88 \\
\hline & K6 & 1.00 & 1.00 & 1.00 & 0.00 & 0.89 \\
\hline \multirow[t]{2}{*}{ K4 } & K5 & 0.99 & 1.00 & 0.92 & 1.00 & 0.08 \\
\hline & K6 & 0.99 & 0.99 & 0.91 & 1.00 & 0.09 \\
\hline K5 & K6 & 1.00 & 1.00 & 1.00 & 0.99 & 1.00 \\
\hline
\end{tabular}

Table SI-11. Significant differences ( $P<0.05$, Tukey's post hocTest) in OCPs concentrations among the sites in Hetauda (only the chemicals with variations have been shown)

\begin{tabular}{cccccc}
\hline & & $\mathrm{p}, \mathrm{p}$-DDE & $\beta-\mathrm{HCH}$ & $\gamma$-HCH & PCBs \\
\hline H1 & H2 & 0.29 & 0.32 & $\mathbf{0 . 0 1}$ & $\mathbf{0 . 0 3}$ \\
& H3 & $\mathbf{0 . 0 1}$ & 0.43 & 1.00 & 0.19 \\
H2 & H3 & 0.20 & $\mathbf{0 . 0 4}$ & 0.01 & 0.53 \\
\hline
\end{tabular}




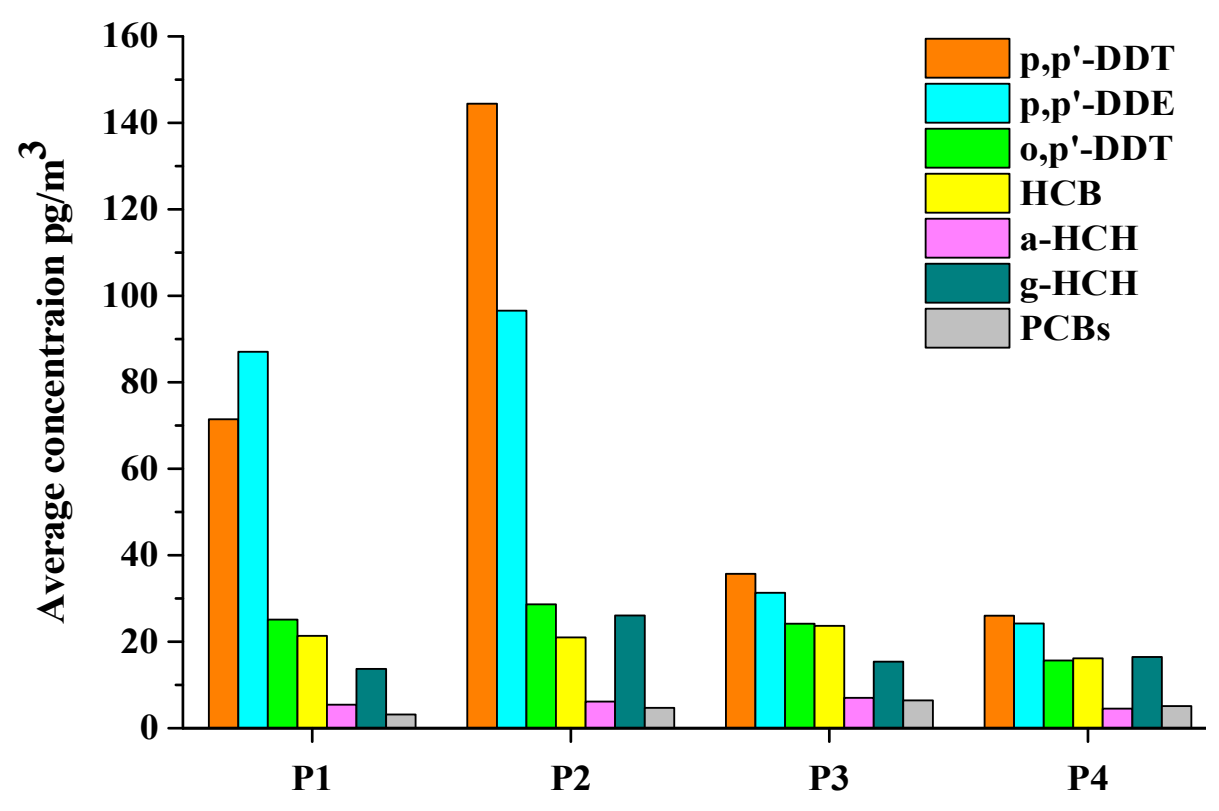

Figure SI-7. Atmospheric level of OCPs in different land cover types in Pokhara; (P1-Cropland; P2-

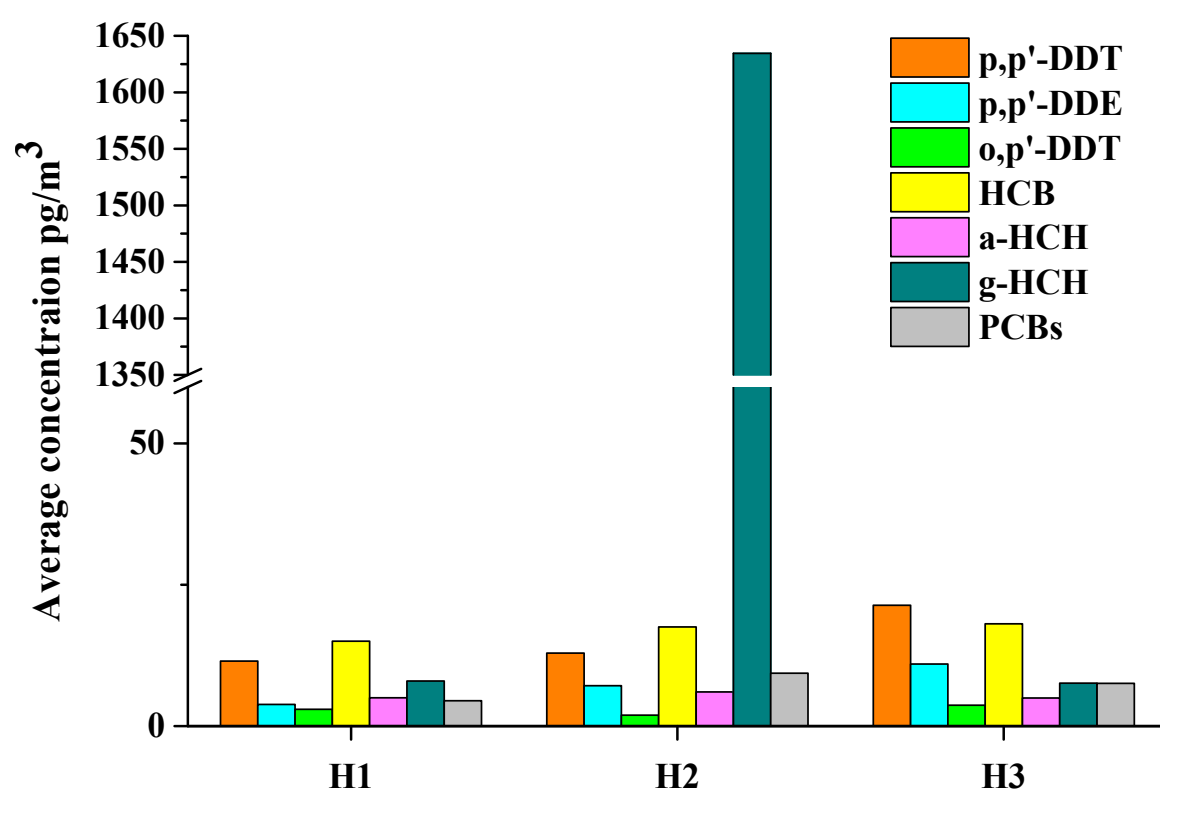

Figure SI-8. Atmospheric level of OCPs in different land cover types in Hetauda; (H1-Crop Land; 
K1
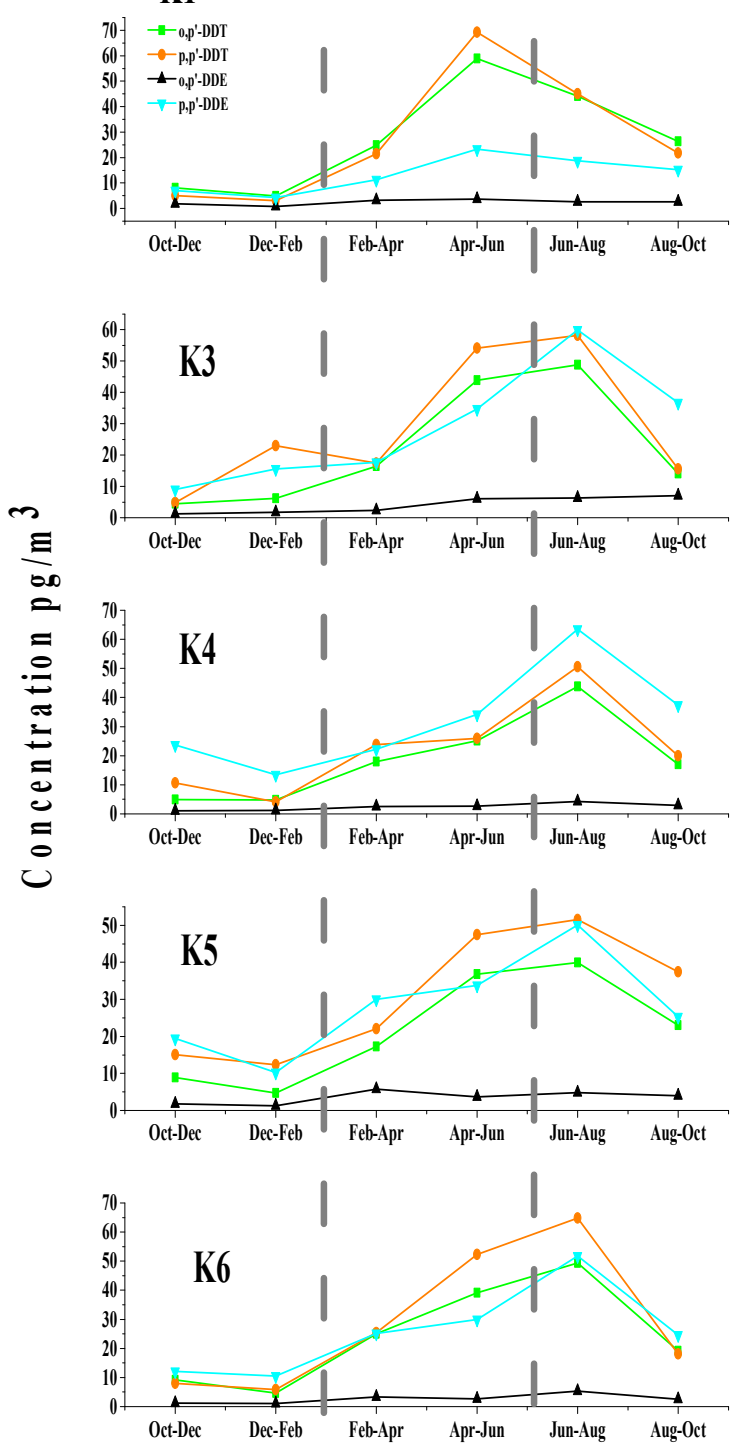

Winter Pre-monsoon Monsoon
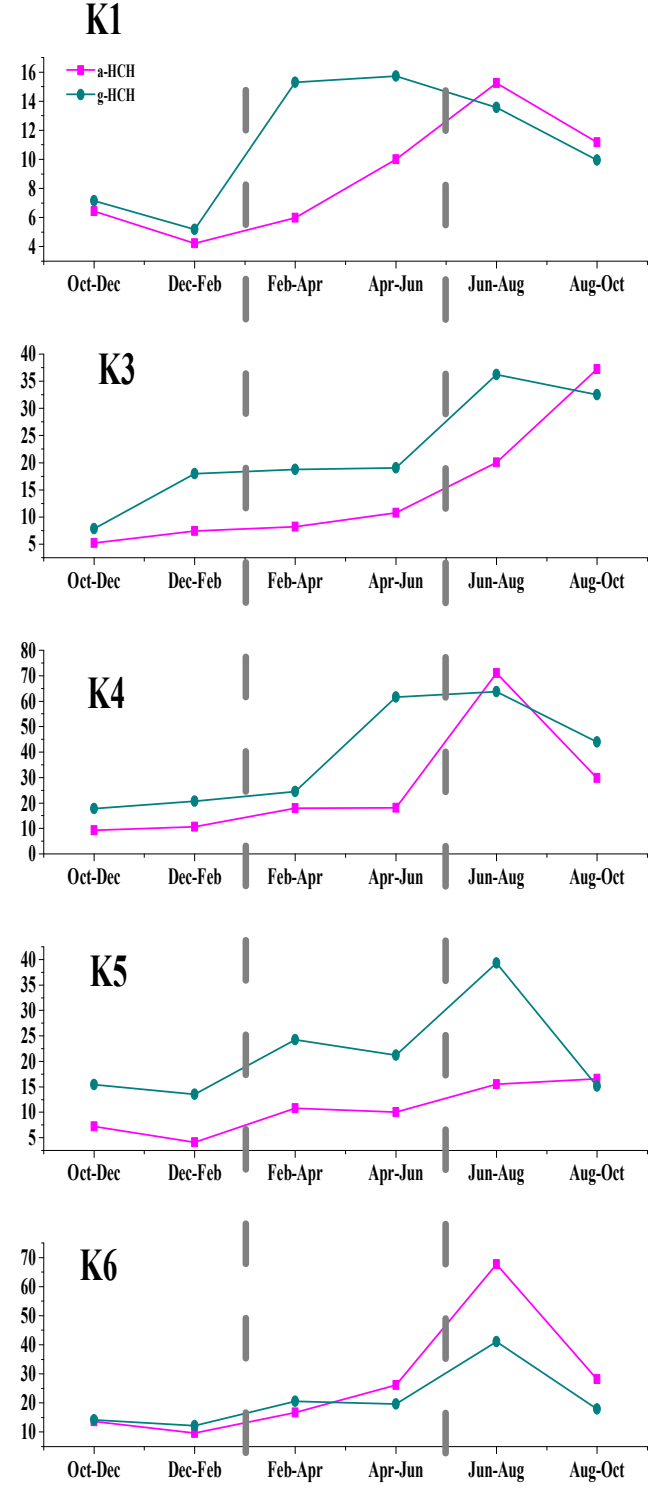

Winter Pre-monsoon Monsoon

Figure SI-9. Seasonality of DDTs and HCHs in Kathmandu city (K1: Cropland, K3: Industrial area, 

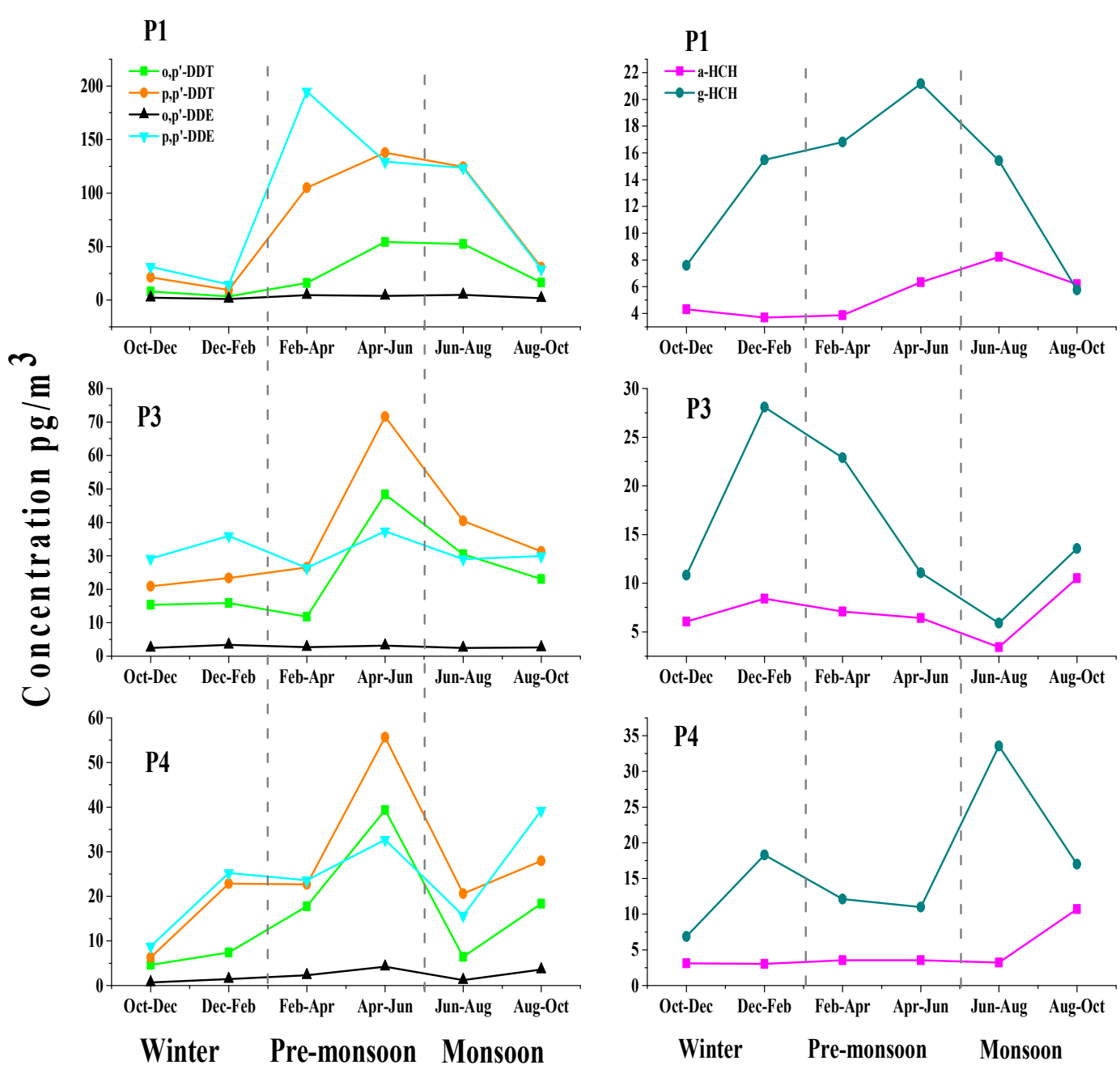

Figure SI-10. Seasonality of DDTs and HCHs in Pokhara city (P1: Cropland, P3: Industrial area, 

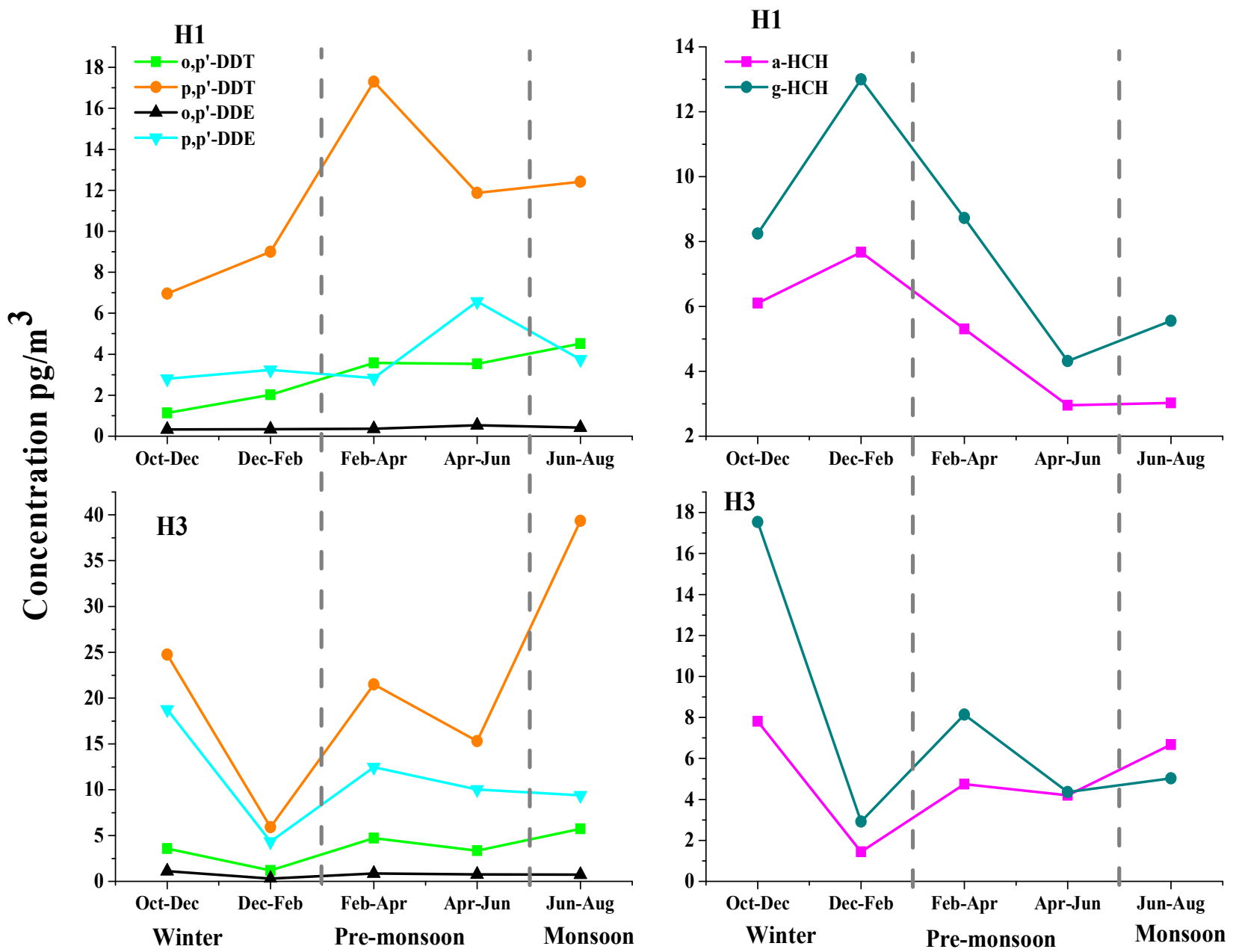

Figure SI-11. Seasonality of DDTs and HCHs in Hetauda (H1: Cropland, H3: Industrial area) 

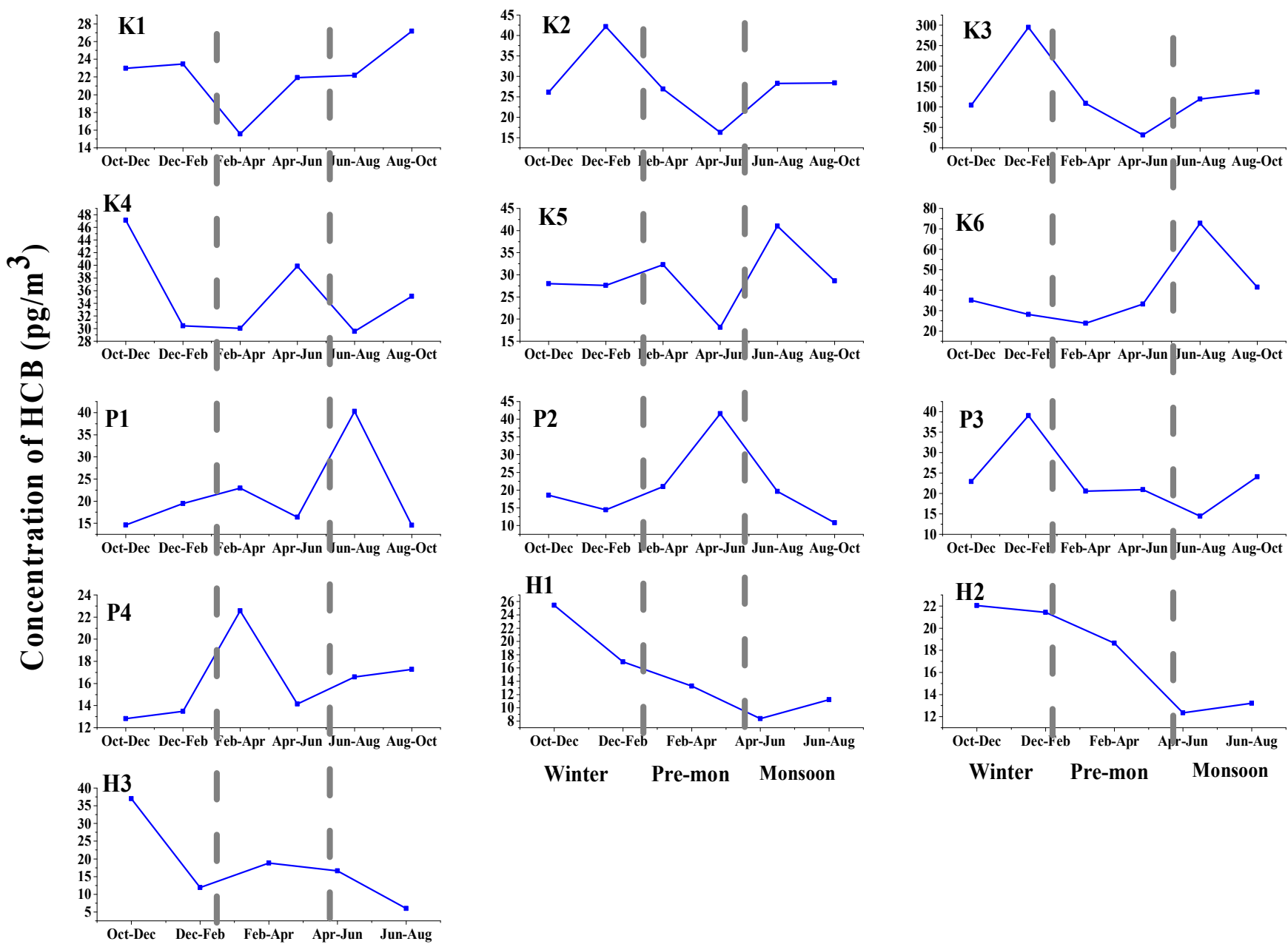

Winter Pre-mon Monsoon

Winter Pre-mon Monsoon

Monsoon 
226 In the equation,

$$
\tau_{a}=\frac{\ln 2}{k_{\text {degr }}+k_{w e t}+k_{d r y}}
$$

$$
\begin{aligned}
& k_{\text {degr }} \text { is photochemical degradation rate in air }\left(\mathrm{s}^{-1}\right) \\
& k_{\text {wet }} \text { wet deposition rate }\left(\mathrm{s}^{-1}\right) \\
& k_{d r y} \text { dry deposition rate }\left(\mathrm{s}^{-1}\right)
\end{aligned}
$$

In general, degradation due to $\mathrm{OH}$ is considered the dominant process and Bayer et al., 2003 derived a simple temperature dependent relation to estimate $\mathrm{OH}$ concentration i.e. $[\mathrm{OH}]$ in atmosphere.

$$
[O H]=0.5+0.4(T-273.15) \times 10^{5}
$$

where $T$ is absolute temperature $(\mathrm{K})$

Then, using the rate constant $\mathrm{K}_{\mathrm{OH}}$ (Table SI-12) the degradation rate $k_{\text {degr }}$ is estimated as,

$$
k_{\text {degr }}=K_{O H}[\mathrm{OH}]
$$

Assuming the gas phase as dominant form of the pollutants in the atmosphere wet deposition has been estimated using the relation

$$
k_{w e t}=\frac{R_{i} W_{G}}{h}
$$

Where $R_{i}=$ annual rain intensity $\left(\mathrm{mm} \mathrm{a}^{-1}\right)$

$W_{G}=$ gas phase scavenging ratio

$h=$ atmospheric boundary layer height (m) and

effective gas phase scavenging ratio is estimated as reciprocal of Henry law coefficient

$$
W_{G}=\frac{R T}{H}
$$

where $R=$ Gas law constant $\left(8.314 \mathrm{~Pa} \mathrm{~m}^{3} \mathrm{~mol}^{-1} \mathrm{~K}^{-1}\right)$

$T=$ absolute temperature $(\mathrm{K})$

$H=$ Henry's law constant

For dry deposition rate the $k_{d r y}$, has been estimated as

$$
k_{d r y}=\frac{V_{D}}{h}
$$

Where $V_{D}$ is dry deposition velocity $\left(\mathrm{cm} \mathrm{s}^{-1}\right)$ 

proposed by González-Gaya et al., 2014

$$
\log V_{D}=-0.261 \log P L-2.670 \mathrm{~cm} \mathrm{~s}^{-1}
$$

261 Table SI-12. Temperature dependent Henry's law constant and vapor pressure with Rate constant 262 of hydroxyl radical reaction at $25^{\circ} \mathrm{C}$

263

\begin{tabular}{|c|c|c|c|}
\hline Compounds & $\log H\left(\mathrm{~Pa} \mathrm{~m}^{3} \mathrm{~mol}^{-1}\right)$ & $\log P L(P a)$ & $K_{O H}\left(25^{\circ} \mathrm{C}\right) \mathrm{cm}^{3} \mathrm{molec}^{-1} \mathrm{~s}^{-1}$ \\
\hline $\mathrm{p}, \mathrm{p}^{\prime}-\mathrm{DDT}$ & $13.02-3369 / \mathrm{T}$ & $13.02-4865 / \mathrm{T}$ & $1.5 \times 10^{-12}$ \\
\hline$\alpha-\mathrm{HCH}$ & $8.98-1714 / \mathrm{T}$ & $11.12-3497 / \mathrm{T}$ & $1.4 \times 10^{-13}$ \\
\hline$\gamma-\mathrm{HCH}$ & $11.58-3049 / \mathrm{T}$ & $11.98-3905 / \mathrm{T}$ & $1.9 \times 10^{-13}$ \\
\hline HCB & $11.6-3013 / \mathrm{T}$ & $11.11-3582 / \mathrm{T}$ & $2.7 \times 10^{-14}$ \\
\hline
\end{tabular}

265 Parameters about Henry's law constants, $\mathrm{OH}$ initiated atmospheric reaction rate and chemical's property data are

266 found from literatures (Passivirta et al., 1999; Hinckley et al., 1990; Bai et al., 2013; Xiao et al., 2004; Brubaker et 267 al., 1998; Jautunen et al., 2006) 


\begin{tabular}{|c|c|c|c|c|c|c|c|c|c|c|c|c|c|}
\hline & \multicolumn{5}{|c|}{$\boldsymbol{k}_{\text {degr }}$} & \multicolumn{4}{|c|}{$\boldsymbol{k}_{w e t}$} & \multicolumn{4}{|c|}{$\boldsymbol{k}_{d r y}$} \\
\hline & Temp & $\mathrm{p}, \mathrm{p}^{\prime}-\mathrm{DDT}$ & $\gamma-\mathrm{HCH}$ & $\alpha-\mathrm{HCH}$ & $\mathrm{HCB}$ & $\mathrm{p}, \mathrm{p}^{\prime}-\mathrm{DDT}$ & $\gamma-\mathrm{HCH}$ & $\alpha-\mathrm{HCH}$ & $\mathrm{HCB}$ & $\mathrm{p}, \mathrm{p}^{\prime}-\mathrm{DDT}$ & $\gamma-\mathrm{HCH}$ & $\alpha-\mathrm{HCH}$ & $\mathrm{HCB}$ \\
\hline \multicolumn{14}{|c|}{ Kathmandu } \\
\hline Jan & 284.15 & $9.66 \mathrm{E}-08$ & $1.22 \mathrm{E}-08$ & $9.02 \mathrm{E}-09$ & 1.74E-09 & $2.48 \mathrm{E}-08$ & $5.12 \mathrm{E}-08$ & $4.08 \mathrm{E}-10$ & $3.65 \mathrm{E}-08$ & 8.38E-07 & $2.06 \mathrm{E}-07$ & $1.45 \mathrm{E}-07$ & $1.75 \mathrm{E}-07$ \\
\hline Feb & 286.15 & $2.23 \mathrm{E}-07$ & $2.82 \mathrm{E}-08$ & $2.08 \mathrm{E}-08$ & 4.01E-09 & 2.07E-08 & 4.34E-08 & $3.73 \mathrm{E}-10$ & $3.10 \mathrm{E}-08$ & $7.80 \mathrm{E}-07$ & $1.94 \mathrm{E}-07$ & $1.38 \mathrm{E}-07$ & $1.66 \mathrm{E}-07$ \\
\hline Mar & 290.15 & 8.52E-07 & $1.08 \mathrm{E}-07$ & 7.95E-08 & $1.53 \mathrm{E}-08$ & $1.44 \mathrm{E}-08$ & $3.13 \mathrm{E}-08$ & $3.13 \mathrm{E}-10$ & $2.25 \mathrm{E}-08$ & $6.77 \mathrm{E}-07$ & $1.73 \mathrm{E}-07$ & $1.25 \mathrm{E}-07$ & $1.50 \mathrm{E}-07$ \\
\hline Apr & 293.15 & $1.92 \mathrm{E}-06$ & $2.43 \mathrm{E}-07$ & $1.79 \mathrm{E}-07$ & $3.46 \mathrm{E}-08$ & $1.11 \mathrm{E}-08$ & $2.47 \mathrm{E}-08$ & $2.75 \mathrm{E}-10$ & $1.78 \mathrm{E}-08$ & $6.11 \mathrm{E}-07$ & $1.60 \mathrm{E}-07$ & $1.16 \mathrm{E}-07$ & $1.39 \mathrm{E}-07$ \\
\hline May & 295.15 & $3.09 \mathrm{E}-06$ & $3.92 \mathrm{E}-07$ & 2.89E-07 & 5.57E-08 & 9.33E-09 & $2.12 \mathrm{E}-08$ & $2.53 \mathrm{E}-10$ & $1.53 \mathrm{E}-08$ & 5.71E-07 & $1.51 \mathrm{E}-07$ & $1.10 \mathrm{E}-07$ & $1.32 \mathrm{E}-07$ \\
\hline Jun & 297.15 & 4.78E-06 & $6.05 \mathrm{E}-07$ & $4.46 \mathrm{E}-07$ & $8.60 \mathrm{E}-08$ & 7.87E-09 & $1.82 \mathrm{E}-08$ & $2.32 \mathrm{E}-10$ & $1.31 \mathrm{E}-08$ & 5.34E-07 & $1.43 \mathrm{E}-07$ & $1.05 \mathrm{E}-07$ & $1.26 \mathrm{E}-07$ \\
\hline Jul & 297.15 & 4.78E-06 & $6.05 \mathrm{E}-07$ & $4.46 \mathrm{E}-07$ & $8.60 \mathrm{E}-08$ & 7.87E-09 & $1.82 \mathrm{E}-08$ & $2.32 \mathrm{E}-10$ & $1.31 \mathrm{E}-08$ & 5.34E-07 & $1.43 \mathrm{E}-07$ & $1.05 \mathrm{E}-07$ & $1.26 \mathrm{E}-07$ \\
\hline Aug & 297.15 & $4.78 \mathrm{E}-06$ & $6.05 \mathrm{E}-07$ & $4.46 \mathrm{E}-07$ & $8.60 \mathrm{E}-08$ & 7.87E-09 & $1.82 \mathrm{E}-08$ & $2.32 \mathrm{E}-10$ & $1.31 \mathrm{E}-08$ & $5.34 \mathrm{E}-07$ & $1.43 \mathrm{E}-07$ & $1.05 \mathrm{E}-07$ & $1.26 \mathrm{E}-07$ \\
\hline Sep & 296.15 & $3.86 \mathrm{E}-06$ & 4.89E-07 & $3.60 \mathrm{E}-07$ & $6.95 \mathrm{E}-08$ & 8.57E-09 & $1.96 \mathrm{E}-08$ & $2.42 \mathrm{E}-10$ & $1.41 \mathrm{E}-08$ & 5.52E-07 & $1.47 \mathrm{E}-07$ & $1.08 \mathrm{E}-07$ & $1.29 \mathrm{E}-07$ \\
\hline Oct & 293.15 & $1.92 \mathrm{E}-06$ & $2.43 \mathrm{E}-07$ & 1.79E-07 & $3.46 \mathrm{E}-08$ & $1.11 \mathrm{E}-08$ & $2.47 \mathrm{E}-08$ & $2.75 \mathrm{E}-10$ & $1.78 \mathrm{E}-08$ & $6.11 \mathrm{E}-07$ & $1.60 \mathrm{E}-07$ & $1.16 \mathrm{E}-07$ & $1.39 \mathrm{E}-07$ \\
\hline Nov & 289.15 & $6.29 \mathrm{E}-07$ & 7.97E-08 & 5.87E-08 & $1.13 \mathrm{E}-08$ & $1.58 \mathrm{E}-08$ & $3.40 \mathrm{E}-08$ & $3.27 \mathrm{E}-10$ & $2.44 \mathrm{E}-08$ & $7.02 \mathrm{E}-07$ & $1.78 \mathrm{E}-07$ & $1.28 \mathrm{E}-07$ & $1.54 \mathrm{E}-07$ \\
\hline Dec & 285.15 & $1.49 \mathrm{E}-07$ & $1.89 \mathrm{E}-08$ & $1.39 \mathrm{E}-08$ & 2.69E-09 & $2.27 \mathrm{E}-08$ & 4.71E-08 & $3.90 \mathrm{E}-10$ & $3.36 \mathrm{E}-08$ & $8.08 \mathrm{E}-07$ & $2.00 \mathrm{E}-07$ & $1.42 \mathrm{E}-07$ & $1.71 \mathrm{E}-07$ \\
\hline \multicolumn{14}{|c|}{ Pokhara } \\
\hline Jan & 286.15 & $2.23 \mathrm{E}-07$ & $2.82 \mathrm{E}-08$ & $2.08 \mathrm{E}-08$ & 4.01E-09 & 5.56E-08 & $1.17 \mathrm{E}-07$ & $1.00 \mathrm{E}-09$ & 8.34E-08 & $7.80 \mathrm{E}-07$ & $1.94 \mathrm{E}-07$ & $1.38 \mathrm{E}-07$ & $1.66 \mathrm{E}-07$ \\
\hline Feb & 289.15 & $6.29 \mathrm{E}-07$ & 7.97E-08 & 5.87E-08 & $1.13 \mathrm{E}-08$ & 4.24E-08 & $9.14 \mathrm{E}-08$ & $8.79 \mathrm{E}-10$ & $6.55 \mathrm{E}-08$ & $7.02 \mathrm{E}-07$ & $1.78 \mathrm{E}-07$ & $1.28 \mathrm{E}-07$ & $1.54 \mathrm{E}-07$ \\
\hline Mar & 293.15 & $1.92 \mathrm{E}-06$ & $2.43 \mathrm{E}-07$ & $1.79 \mathrm{E}-07$ & $3.46 \mathrm{E}-08$ & $2.98 \mathrm{E}-08$ & $6.65 \mathrm{E}-08$ & $7.40 \mathrm{E}-10$ & 4.79E-08 & $6.11 \mathrm{E}-07$ & $1.60 \mathrm{E}-07$ & $1.16 \mathrm{E}-07$ & $1.39 \mathrm{E}-07$ \\
\hline Apr & 296.15 & $3.86 \mathrm{E}-06$ & 4.89E-07 & $3.60 \mathrm{E}-07$ & $6.95 \mathrm{E}-08$ & $2.31 \mathrm{E}-08$ & $5.27 \mathrm{E}-08$ & $6.52 \mathrm{E}-10$ & $3.81 \mathrm{E}-08$ & $5.52 \mathrm{E}-07$ & $1.47 \mathrm{E}-07$ & $1.08 \mathrm{E}-07$ & $1.29 \mathrm{E}-07$ \\
\hline May & 297.15 & $4.78 \mathrm{E}-06$ & $6.05 \mathrm{E}-07$ & $4.46 \mathrm{E}-07$ & 8.60E-08 & 2.12E-08 & 4.89E-08 & $6.26 \mathrm{E}-10$ & $3.53 \mathrm{E}-08$ & 5.34E-07 & $1.43 \mathrm{E}-07$ & $1.05 \mathrm{E}-07$ & $1.26 \mathrm{E}-07$ \\
\hline
\end{tabular}




\begin{tabular}{|c|c|c|c|c|c|c|c|c|c|c|c|c|c|}
\hline Jun & 299.15 & 7.13E-06 & $9.03 \mathrm{E}-07$ & $6.65 \mathrm{E}-07$ & $1.28 \mathrm{E}-07$ & $1.79 \mathrm{E}-08$ & 4.20E-08 & $5.76 \mathrm{E}-10$ & 3.04E-08 & $5.00 \mathrm{E}-07$ & $1.36 \mathrm{E}-07$ & $1.00 \mathrm{E}-07$ & $1.20 \mathrm{E}-07$ \\
\hline Jul & 299.15 & 7.13E-06 & $9.03 \mathrm{E}-07$ & $6.65 \mathrm{E}-07$ & $1.28 \mathrm{E}-07$ & $1.79 \mathrm{E}-08$ & 4.20E-08 & $5.76 \mathrm{E}-10$ & 3.04E-08 & $5.00 \mathrm{E}-07$ & $1.36 \mathrm{E}-07$ & $1.00 \mathrm{E}-07$ & $1.20 \mathrm{E}-07$ \\
\hline Aug & 299.15 & 7.13E-06 & $9.03 \mathrm{E}-07$ & $6.65 \mathrm{E}-07$ & $1.28 \mathrm{E}-07$ & $1.79 \mathrm{E}-08$ & $4.20 \mathrm{E}-08$ & $5.76 \mathrm{E}-10$ & $3.04 \mathrm{E}-08$ & $5.00 \mathrm{E}-07$ & $1.36 \mathrm{E}-07$ & $1.00 \mathrm{E}-07$ & $1.20 \mathrm{E}-07$ \\
\hline Sep & 298.15 & $5.86 \mathrm{E}-06$ & $7.42 \mathrm{E}-07$ & $5.47 \mathrm{E}-07$ & $1.05 \mathrm{E}-07$ & $1.95 \mathrm{E}-08$ & $4.53 \mathrm{E}-08$ & $6.00 \mathrm{E}-10$ & $3.28 \mathrm{E}-08$ & $5.17 \mathrm{E}-07$ & $1.39 \mathrm{E}-07$ & $1.03 \mathrm{E}-07$ & $1.23 \mathrm{E}-07$ \\
\hline Oct & 295.15 & 3.09E-06 & 3.92E-07 & $2.89 \mathrm{E}-07$ & $5.57 \mathrm{E}-08$ & $2.51 \mathrm{E}-08$ & $5.70 \mathrm{E}-08$ & $6.80 \mathrm{E}-10$ & 4.11E-08 & $5.71 \mathrm{E}-07$ & $1.51 \mathrm{E}-07$ & $1.10 \mathrm{E}-07$ & $1.32 \mathrm{E}-07$ \\
\hline Nov & 291.15 & $1.13 \mathrm{E}-06$ & $1.44 \mathrm{E}-07$ & $1.06 \mathrm{E}-07$ & $2.04 \mathrm{E}-08$ & $3.55 \mathrm{E}-08$ & 7.79E-08 & $8.06 \mathrm{E}-10$ & $5.60 \mathrm{E}-08$ & $6.54 \mathrm{E}-07$ & $1.69 \mathrm{E}-07$ & $1.22 \mathrm{E}-07$ & $1.46 \mathrm{E}-07$ \\
\hline Dec & 287.15 & $3.23 \mathrm{E}-07$ & 4.09E-08 & $3.01 \mathrm{E}-08$ & 5.81E-09 & $5.08 \mathrm{E}-08$ & $1.08 \mathrm{E}-07$ & $9.60 \mathrm{E}-10$ & 7.69E-08 & $7.53 \mathrm{E}-07$ & $1.89 \mathrm{E}-07$ & $1.35 \mathrm{E}-07$ & $1.62 \mathrm{E}-07$ \\
\hline \multicolumn{14}{|c|}{ Hetauda } \\
\hline Jan & 288.15 & $4.56 \mathrm{E}-07$ & 5.77E-08 & $4.25 \mathrm{E}-08$ & 8.20E-09 & $2.68 \mathrm{E}-08$ & $5.71 \mathrm{E}-08$ & $5.30 \mathrm{E}-10$ & 4.09E-08 & 7.27E-07 & $1.83 \mathrm{E}-07$ & $1.31 \mathrm{E}-07$ & $1.58 \mathrm{E}-07$ \\
\hline Feb & 290.15 & $8.52 \mathrm{E}-07$ & $1.08 \mathrm{E}-07$ & 7.95E-08 & $1.53 \mathrm{E}-08$ & $2.24 \mathrm{E}-08$ & 4.86E-08 & $4.85 \mathrm{E}-10$ & $3.49 \mathrm{E}-08$ & $6.77 \mathrm{E}-07$ & $1.73 \mathrm{E}-07$ & $1.25 \mathrm{E}-07$ & $1.50 \mathrm{E}-07$ \\
\hline Mar & 294.15 & $2.45 \mathrm{E}-06$ & $3.10 \mathrm{E}-07$ & $2.29 \mathrm{E}-07$ & 4.41E-08 & $1.58 \mathrm{E}-08$ & $3.55 \mathrm{E}-08$ & $4.09 \mathrm{E}-10$ & $2.56 \mathrm{E}-08$ & 5.91E-07 & $1.55 \mathrm{E}-07$ & $1.13 \mathrm{E}-07$ & $1.35 \mathrm{E}-07$ \\
\hline Apr & 299.15 & 7.13E-06 & $9.03 \mathrm{E}-07$ & $6.65 \mathrm{E}-07$ & $1.28 \mathrm{E}-07$ & $1.03 \mathrm{E}-08$ & $2.42 \mathrm{E}-08$ & $3.32 \mathrm{E}-10$ & $1.75 \mathrm{E}-08$ & $5.00 \mathrm{E}-07$ & $1.36 \mathrm{E}-07$ & $1.00 \mathrm{E}-07$ & $1.20 \mathrm{E}-07$ \\
\hline May & 301.15 & $1.03 \mathrm{E}-05$ & $1.31 \mathrm{E}-06$ & $9.64 \mathrm{E}-07$ & $1.86 \mathrm{E}-07$ & $8.75 \mathrm{E}-09$ & 2.09E-08 & $3.06 \mathrm{E}-10$ & $1.51 \mathrm{E}-08$ & 4.69E-07 & $1.29 \mathrm{E}-07$ & $9.58 \mathrm{E}-08$ & $1.14 \mathrm{E}-07$ \\
\hline Jun & 301.15 & $1.03 \mathrm{E}-05$ & $1.31 \mathrm{E}-06$ & $9.64 \mathrm{E}-07$ & $1.86 \mathrm{E}-07$ & $8.75 \mathrm{E}-09$ & 2.09E-08 & $3.06 \mathrm{E}-10$ & $1.51 \mathrm{E}-08$ & 4.69E-07 & $1.29 \mathrm{E}-07$ & $9.58 \mathrm{E}-08$ & $1.14 \mathrm{E}-07$ \\
\hline Jul & 300.15 & 8.61E-06 & 1.09E-06 & 8.04E-07 & $1.55 \mathrm{E}-07$ & $9.50 \mathrm{E}-09$ & $2.25 \mathrm{E}-08$ & $3.19 \mathrm{E}-10$ & $1.63 \mathrm{E}-08$ & 4.84E-07 & $1.32 \mathrm{E}-07$ & $9.80 \mathrm{E}-08$ & $1.17 \mathrm{E}-07$ \\
\hline Aug & 300.15 & 8.61E-06 & $1.09 \mathrm{E}-06$ & 8.04E-07 & $1.55 \mathrm{E}-07$ & $9.50 \mathrm{E}-09$ & $2.25 \mathrm{E}-08$ & $3.19 \mathrm{E}-10$ & $1.63 \mathrm{E}-08$ & 4.84E-07 & $1.32 \mathrm{E}-07$ & $9.80 \mathrm{E}-08$ & $1.17 \mathrm{E}-07$ \\
\hline Sep & 299.15 & 7.13E-06 & $9.03 \mathrm{E}-07$ & $6.65 \mathrm{E}-07$ & $1.28 \mathrm{E}-07$ & $1.03 \mathrm{E}-08$ & $2.42 \mathrm{E}-08$ & $3.32 \mathrm{E}-10$ & $1.75 \mathrm{E}-08$ & $5.00 \mathrm{E}-07$ & $1.36 \mathrm{E}-07$ & $1.00 \mathrm{E}-07$ & $1.20 \mathrm{E}-07$ \\
\hline Oct & 298.15 & $5.86 \mathrm{E}-06$ & 7.42E-07 & $5.47 \mathrm{E}-07$ & $1.05 \mathrm{E}-07$ & $1.12 \mathrm{E}-08$ & $2.61 \mathrm{E}-08$ & $3.46 \mathrm{E}-10$ & $1.89 \mathrm{E}-08$ & $5.17 \mathrm{E}-07$ & $1.39 \mathrm{E}-07$ & $1.03 \mathrm{E}-07$ & $1.23 \mathrm{E}-07$ \\
\hline Nov & 293.15 & $1.92 \mathrm{E}-06$ & $2.43 \mathrm{E}-07$ & 1.79E-07 & $3.46 \mathrm{E}-08$ & $1.72 \mathrm{E}-08$ & $3.84 \mathrm{E}-08$ & $4.27 \mathrm{E}-10$ & $2.76 \mathrm{E}-08$ & $6.11 \mathrm{E}-07$ & $1.60 \mathrm{E}-07$ & $1.16 \mathrm{E}-07$ & $1.39 \mathrm{E}-07$ \\
\hline Dec & 288.15 & $4.56 \mathrm{E}-07$ & $5.77 \mathrm{E}-08$ & 4.25E-08 & 8.20E-09 & $2.68 \mathrm{E}-08$ & $5.71 \mathrm{E}-08$ & $5.30 \mathrm{E}-10$ & 4.09E-08 & $7.27 \mathrm{E}-07$ & $1.83 \mathrm{E}-07$ & $1.31 \mathrm{E}-07$ & $1.58 \mathrm{E}-07$ \\
\hline
\end{tabular}


273 Table SI-14. Comparison of characteristic travel distance (CTD, $\mathrm{km})$ in current study areas 274 with global and other specified regions

275

\begin{tabular}{|c|c|c|c|c|c|c|c|c|c|}
\hline & \multicolumn{4}{|c|}{ This study (km) } & \multicolumn{5}{|c|}{ Previous studies } \\
\hline & Kathmandu & Pokhar & Hetauda & average & $\begin{array}{c}* \text { Global } \\
\text { (a) }\end{array}$ & $\begin{array}{c}* \text { Global } \\
\text { (b) }\end{array}$ & $\begin{array}{c}\text { East \& } \\
\text { south china } \\
\text { seas } * *\end{array}$ & $\begin{array}{c}\text { Indian } \\
\text { Ocean** }\end{array}$ & $\begin{array}{c}\text { South } \\
\text { Atlantic } \\
* *\end{array}$ \\
\hline $\mathrm{HCB}$ & 11836 & 9834 & 9984 & 10551 & 10600 & 144304 & 13306 & 345 & 907 \\
\hline$\alpha-\mathrm{HCH}$ & 9346 & 7536 & 6250 & 7710 & 17946 & 22307 & 3629 & 605 & 484 \\
\hline$\gamma-\mathrm{HCH}$ & 6016 & 4387 & 4035 & 4812 & 9732 & 22572 & 3024 & 544 & 363 \\
\hline $\begin{array}{l}\mathrm{p}, \mathrm{p}^{\prime}- \\
\text { DDT }\end{array}$ & 1269 & 956 & 776 & 1000 & 1045 & 1462 & 1331 & 774 & \\
\hline
\end{tabular}

*Shen et al., 2005 ( $a$ : estimation by TaPL3 model; b: estimation by ELPOS)

** Gioia et al., 2012 
279 Given soil can absorb atmospheric POPs, it may retard the transport of POPs. Previous study had 280 investigated the air-soil exchange of POPs along south slope of Nepal Himalaya mountain, and the 281 results found volatile compounds such as HCB, PCBs and HCHs reached air-soil exchange equilibrium in low elevation cities (Gong et al., 2014). This means for the volatile compounds, only the atmospheric processes (i.e. $\mathrm{OH}$ degradation and dry/wet deposition) are major loss process. Due to the possible overestimation of $\mathrm{OH}$ concentration reported by Anderson et al. (1996), $\tau_{a}$ will be underestimated and then CTD will be underestimated. With respect to less volatile compounds such as DDTs, their air-soil exchange showed deposition trend (Gong et al., 2014), suggesting soil absorption should not be ignored. As this will reduced $\tau_{a}$ of DDTs, and lead to the overestimation of CTD.

\section{Text SI-7 Generation of forward trajectories}

NOAA's HYSPLIT model and the NCEP/NCAR Global Reanalysis data set for Kathmandu were used to calculate forward trajectories. Forward trajectories were traced for 3 days at $6 \mathrm{~h}$ intervals at $100 \mathrm{~m}$ above sea level. All 416 trajectories were grouped into 5 clusters. Sixty present of trajectories (sum of cluster 1, 2 and 3) move northward, crossing the Himalaya and reaching southeastern Tibetan Plateau.

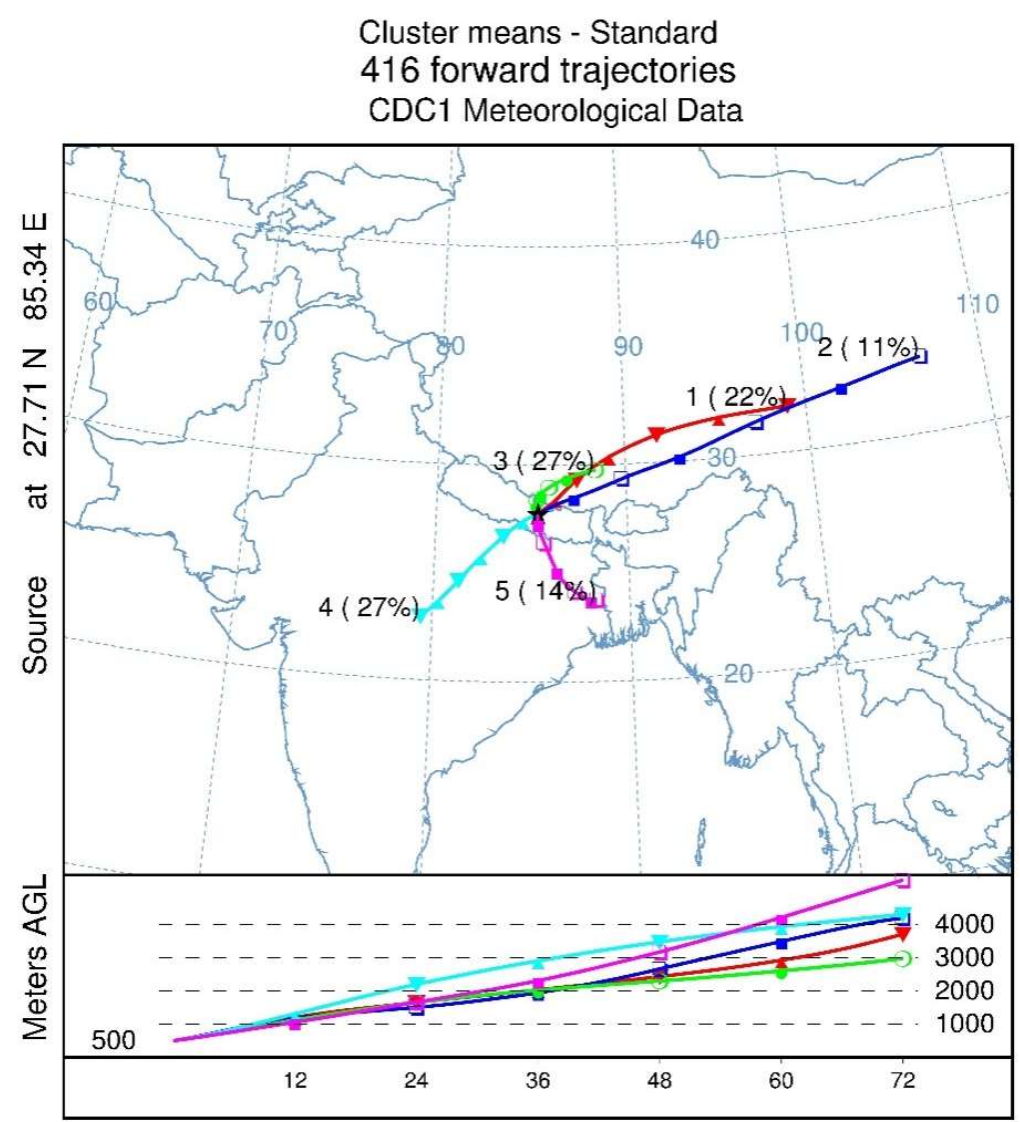




\section{References:}

Anderson, P. N.; Hites., R. A. OH radical reactions: The major removal pathway for polychlorinated biphenyls from the atmosphere. Environ. Sci. Technol. 1996, 30, 1757-1763

Bai, J.; Yang, W.; Zhang, C.; Zhao, Y.; Gong, C.; Sun, X.; Zhang, Q.; Wang, W. Theoretical study on the OH-initiated atmospheric reaction of 1,1,1-trichloro-2,2-bis(4-chlorophenyl) ethane (DDT). Atmos. Environ. 2013, 67, 177183.

Beyer, A.; Wania, F.; Gouin, T.; Mackay, D.; Matthies, M. Temperature dependence of the characteristic travel distance. Environ. Sci. Technol.2003, 37 (4), 766-771.

Brubaker, W. W.; Hites, R. A. OH Reaction Kinetics of Gas-Phase a- and $\gamma$-Hexachlorocyclohexane and Hexachlorobenzene. Environ. Sci. Technol.1998, 32 (6), 766-769.

Gioia, R.; Li, J.; Schuster, J.; Zhang, Y.; Zhang, G.; Li, X.; h Spiro, B.; Bhatia, R. S., Dachs, J.; Jones, K.C. Factors Affecting the Occurrence and Transport of Atmospheric Organochlorines in the China Sea and the Northern Indian and South East Atlantic Oceans. Environ. Sci. Technol. 2012, 46, 10012-10021

Gong, P.; Wang, X.; Li, S.; Yu, W.; Li, J.; Kattel, D. B.; Wang, W.; Deykota, L. P.; Yao, T.D.; Joswiak, D. R. Atmospheric transport and accumulation of organochlorine compounds on the southern slopes of the Himalayas, Nepal Environ. Pollut. 2014, 192, 44-51.

González-Gaya, B.; Zúñiga-Rival, J.; Ojeda, M. J.; Jiménez, B.; Dachs, J. Field measurements of the atmospheric dry deposition fluxes and velocities of polycyclic aromatic hydrocarbons to the global oceans. Environ. Sci. Technol.2014, 48 (10), 5583-5592.

Harner, T.; Shoeib, M.; Diamond, M. L.; Stern, G.; Rosenberg, B. Using passive air samplers to assess urban-rural trends for persistent organic pollutants. 1. Polychlorinated biphenyls and organochlorine pesticides. Environ. Sci. Technol.2004, 38 (17), 4474-4483.

Harner, T.; Bartkow, M.; Holoubek, I.; Klanova, J.; Wania, F.; Gioia, R.; Moeckel, C.; Sweetman, A. J.; Jones, K. C. Passive air sampling for persistent organic pollutants : Introductory remarks to the special issue. Environ. Pollut. 2006, 144, 361-364

Hinckley, D.; TF, B.; ForemanWT; JR., T. Determination of vapor pressures for nonpolar and semipolar organic compounds from gas chromatographic retention data. J Chem Eng Data 1990, 35, 232-237.

Jantunen, L. M.; Bidleman, T. F. Henry's law constants for hexachlorobenzene, p,p'-DDE and components of technical chlordane and estimates of gas exchange for Lake Ontario. Chemosphere 2006, 62 (10), 1689-1696.

Moeckel, C.; Harner, T.; Nizzetto, L.; Strandberg, B.; Lindroth, A.; Jones, K. C. Use of depuration compounds in passive air samplers: Results from active sampling-supported field deployment, potential uses, and recommendations. Environ. Sci. Technol.2009, 43 (9), 3227-3232.

Paasivirta, J.; Sinkkonen, S.; Mikkelson, P.; Rantio, T.; Wania, F. Estimation of vapor pressures, solubilities and Henry's law constants of selected persistent organic pollutants as functions of temperature. Chemosphere 1999, $39(5), 811-832$.

Pozo, K.; Harner, T.; Wania, F.; Muir, D. C. G.; Jones, K. C.; Barrie, L. a. Toward a global network for persistent organic pollutants in air: Results from the GAPS study. Environ. Sci. Technol.2006, 40 (16), 4867-4873.

Pozo, K.; Harner, T.; Lee, S. C.; Wania, F.; Muir, D. C. G.; Jones, K. C. Seasonally Resolved Concentrations of Persistent Organic Pollutants in the Global Atmosphere from the First Year of the GAPS Study. Environ. Sci. Technol.2009, 43 (3), 796-803. 
Sheng, L.; Wania, F.; Lei, Y.; Muir D.C.G.; Teixeira, C.; Bidleman, T.F. Atmospheric Distribution and Long-Range Transport Behavior of Organochlorine Pesticides in North America. Environ. Sci. Technol. 2005, 39, 409-420

Shoeib, M; Haner, T. Characterization and comparision of three passive air samplers for persitent organic pollutants. Environ Dci. Technol. 2002, 36, 4142-4151

Xiao, H.; Li, N.; Wania, F. Compilation, Evaluation , and Selection of Physical-Chemical Property Data for a -, $\beta$ -, $\gamma$ - Hexachlorocyclohexane. J. Chem. Eng. Data 2004, 49, 173-185

Wong, F.; Alegria, H. a; Bidleman, T. F.; Alvarado, V.; Angeles, F.; Galarza, A. a; Bandala, E. R.; De, I.; Hinojosa, C.; Estrada, G.; et al. Passive Air Sampling of Organochlorine Pesticides in Mexico. Environ. Sci. Technol.2009, 43 (3), 704-710. 